TITLE:

\title{
Local gas transfer rate through the free surface in spatially accelerated open-channel turbulence
}

\author{
$\operatorname{AUTHOR}(\mathrm{S})$ :
}

Sanjou, M.

\section{CITATION:}

Sanjou, M.. Local gas transfer rate through the free surface in spatially accelerated open-channel turbulence. Physics of Fluids 2020, 32(10): 105103.

ISSUE DATE:

2020-10

URL:

http://hdl.handle.net/2433/255880

\section{RIGHT:}

This article may be downloaded for personal use only. Any other use requires prior permission of the author and AIP Publishing. This article appeared in Physics of Fluids 32 105103 (2020) and may be found at https://doi.org/10.1063/5.0021098.; The full-text file will be made open to the public on 1 October 2021 in accordance with publisher's 'Terms and Conditions for Self-Archiving'. 


\section{Local gas transfer rate through the free surface in spatially accelerated open- channel turbulence}

Cite as: Phys. Fluids 32, 105103 (2020); https://doi.org/10.1063/5.0021098

Submitted: 07 July 2020 . Accepted: 10 September 2020 . Published Online: 01 October 2020

M. Sanjou

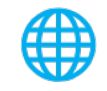

\section{ARTICLES YOU MAY BE INTERESTED IN}

An alternative phase-field interfacial tension force representation for binary fluid systems Physics of Fluids 32, 102101 (2020); https://doi.org/10.1063/5.0026195

On the use of two-point velocity correlation in wall-pressure models for turbulent flow past a trailing edge under adverse pressure gradient

Physics of Fluids 32, 105105 (2020); https://doi.org/10.1063/5.0021121

Three-dimensional deflecting oscillation of turbulent planar opposed jets confined in an open cavity under crossflow

Physics of Fluids 32, 105101 (2020); https://doi.org/10.1063/5.0021501

\section{AIP Advances Fluids and Plasmas Collection}

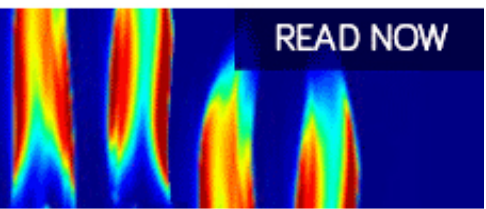




\title{
Local gas transfer rate through the free surface in spatially accelerated open-channel turbulence
}

Cite as: Phys. Fluids 32, 105103 (2020); doi: 10.1063/5.0021098

Submitted: 7 July 2020 - Accepted: 10 September 2020 •

Published Online: 1 October 2020

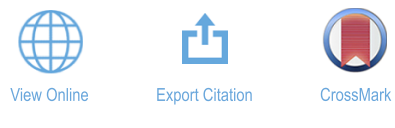

\section{Sanjou}

\author{
AFFILIATIONS \\ Department of Civil and Earth Resources Engineering, Kyoto University, Kyoto 6158540, Japan
}

a) Author to whom correspondence should be addressed: michio.sanjou@water.kuciv.kyoto-u.ac.jp

\begin{abstract}
It is important to determine the transport mechanisms of dissolved gases through free surfaces in open channels. Even though many physical and phenomenological models have been proposed, not much is known about the local distribution of the gas transfer velocity. Thus, this study formulated a theoretical equation for developing a concentration boundary layer in an open-channel flow. Additionally, the free-surface velocity component and the concentration of dissolved oxygen were measured in a spatially accelerated open-channel flow. In particular, the streamwise profile of concentration boundary layer thickness was measured using an extra-fine needle-type dissolved oxygen probe, and the local gas transfer rate was experimentally obtained. The present theory suggests that the local gas transfer is controlled by two significant terms: the streamwise gradient of the mean velocity and the relative intensity of turbulent diffusion. By focusing on accelerated open-channel flows with bottom-situated wedges, the formation mechanism of the concentration boundary layer was explained by a comparison with the presented theory. The comparison of the theoretical model and measurement data indicated that the contributions of both the mean velocity and turbulence diffusion are comparable and significant in the acceleration zone. The thickness of the oxygen concentration boundary layer began to decrease a bit downstream of the entrance, corresponding to the variation in the free-surface turbulence factors. The inflectional point of the concentration thickness appeared downstream of the acceleration zone and continued to decrease at the exit related to relaminarization. These interesting features are explained by the streamwise profiles of the terms in the presented theory.
\end{abstract}

Published under license by AIP Publishing. https://doi.org/10.1063/5.0021098

\section{INTRODUCTION}

\section{A. Background}

It is extremely important to accurately measure and predict oxygen gas transport beneath free surfaces in open-channel flows to properly manage water bodies such as natural rivers, filtration ponds in purification plant, and raceway tanks in aquaculture. Many previous studies have proposed challenging techniques for measuring the reaeration coefficient, for example, the gas tracer method. ${ }^{1}$ The floating chamber method developed by Copeland and Duffer ${ }^{2}$ can sense the oxygen transport flux at the air-water interface within a plastic dome capturing air near the free surface. Even though this permits a direct measurement of the gas flux, the dome itself influences the temperature and pressure and disturbs the water current in the sampling region. ${ }^{3}$ The above-mentioned methods require complicated setups, which result in practical disadvantages. The delta method proposed by Chapra and Di Toro ${ }^{4}$ and McBride and Chapra ${ }^{5}$ measures the day-time dissolved oxygen (DO) concentration in natural aquatic fields and evaluates the reaeration coefficient using the time gap between noon and the time of the observed minimum DO. However, this method is limited to very slow and mild flow conditions. Sanjou et al. ${ }^{6}$ conducted DO sampling in a natural embayment using standard electrode-type probes and obtained the inverse DO concentration slope, which leads to the residence time and the non-dimensional mass-exchange coefficient.

Conversely, in a laboratory recirculating flume and closed tank, we can evaluate the reaeration coefficient using the recovery speed from the initially degassed stage. This is called the "whole tank method" and is often applied to calculations of the gas transfer velocity in the laboratory. Note that the obtained value is the spatially averaged gas transfer velocity, and therefore the whole tank method cannot measure the local gas transfer velocity. In the 21 st century, with the innovative development of high-performance sensing techniques, additional methods have been proposed. For example, Morse et al. ${ }^{7}$ showed that the reaeration coefficient can be reasonably evaluated using the sound pressure. Herlina and Jirka ${ }^{8}$ measured the 
time series of the dissolved gas concentration and instantaneous flow velocity vectors and evaluated the gas transfer flux by applying a vortex correlation method. Variano and Cowen ${ }^{9}$ evaluated the depth-dependent turbulent diffusivity by measuring the scalarvelocity correlation in a stirred laboratory tank. Note that these methods require very accurate calibration procedures.

The basic concept of popular physical models of gas transfer is that the gas flux beneath a free surface is governed by the renewal rate of the fluid parcels near the free surface. These gas transfer models have been studied in experiments and numerical simulations of oscillation tanks and closed channels with wind shear. In contrast, practical studies have focused on the prediction of the reaeration coefficient in natural rivers using the mean velocity, depth, and bottom slope (e.g., Ref. 10). However, there is no universal formula that can be used everywhere because the effects of the turbulent contributions are not sufficiently included. Turbulence generated by a bottom-velocity shear is expected to influence the surface-renewal rate in open-channel flows. ${ }^{11}$ Jackson $^{12}$ described the basic features of a bottom-oriented kolk-boil vortex rising upward, which diverges horizontally in the free surface of an open channel. Therefore, bursting phenomena, such as ejections and sweeps, contribute significantly to the gas transfer. Experimental work by Komori et al. ${ }^{1 .}$ implied that the turbulent structures near the free surface in openchannel flow and large energy-containing vortices control the scalar transfer replacing fluid parcels under the free surface. Using particle image velocimetry (PIV) measurements, Kumar et al. ${ }^{14}$ also concluded that upwelling events near the free surface are significantly influenced by bottom-oriented ejections. Handler et al. ${ }^{15}$ conducted direct numerical simulations of wave-less open-channel flow and obtained similar results to Komori et al. ${ }^{13}$ concerning the anisotropic mechanism near the free-surface turbulence. That is to say, the pressure-strain term of the spanwise turbulent energy becomes a major energy producer and results in a greater increase rate in the spanwise turbulent component near the free surface compared with that in the streamwise turbulent component. The results computed by Nagaosa ${ }^{16}$ suggest that longitudinal tube-like vortices contribute to the near-surface scalar transport via splat/anti-splat motions, and he concluded that scalar transfer is not expected to be promoted by surface-attached vortices related to horizontal swirling on the free surface. Vertical vortices near the free surface tend to lower the free surface, accompanied by some air entrainment. Such a free-surface deformation can be explained using a diagram of the turbulent velocity and length scales. ${ }^{17}$ Chickadel et al. ${ }^{18}$ measured surface eruptions related to meter-sized boils in a tidal river and was able to predict the boil eruption location from measurements of the boil diameter and water depth. Mandel et al. ${ }^{19}$ conducted laboratory experiments in an open channel with submerged cylinders and captured the boil structures derived from the upwelling turbulence.

In addition, some researchers have tried to investigate such detailed mechanisms in open-channel turbulent flows. In laboratory experiments, Gulliver and Halverson ${ }^{20}$ determined the contributions of bursting structures composed of ejections and sweeps to the promotion of the gas transfer rate. Experimental work in openchannel flows by Moog and Jirka ${ }^{21}$ supports a small-eddy model in which the gas transfer is controlled by a small-scale static turbulence parameter such as the dissipation rate. Some numerical and experimental studies have revealed a significant relationship between the mass transfer velocity and the surface divergence (SD) based on the
SD model of McCready et al., ${ }^{22}$ Tamburrino and Gulliver, ${ }^{23}$ Banerjee et al., ${ }^{24}$ Magnoudet and Calmet, ${ }^{25}$ Sanjou et al., ${ }^{26}$ and Leman $e t$ al. $^{27}$ Szeri $^{28}$ developed a new method to determine the gas flux using infrared images. Tseng and Tinoco ${ }^{29}$ proposed a modified surface renewal model for vegetated open-channel flows.

Most previous studies have addressed uniform flow conditions, and therefore, not much is known about the relationship between the local gas transfer velocity and the longitudinal variations in open-channel streams such as natural rivers. The present work is applicable to local-scale based environmental management in natural rivers. The gas transfer velocity or reaeration coefficient is formulated for many rivers. However, most of them are macroscale based empirical models used for large-scale river segment. It is very important to locally evaluate the aquatic environment of rivers because a river is generally composed of various topographies such as meandering, sedimentation/scoring, riffles, and pools. The hydrodynamics and flow pattern are driven significantly by the local topography. Of course, such a non-uniform flow field has a peculiar mass exchange and related specific aquatic ecosystem. When the relationship between the velocity distribution and the gas transfer velocity is found locally, a reasonable prediction of river environment will be possible. These backgrounds motivate the present study.

The present study is expected to be an opportunity for other researchers and engineers to consider new approaches to the local gas transfer problem including numerical simulation. Furthermore, the field observation of the gas transfer velocity is very hard. The method newly suggested here is expected to capture the local concentration boundary layer thickness (CBT) in the rivers, which is formed in the DO recovering process in the early morning. In the same way, this method is applicable to the DO management in the raceway tank used for fisheries. The risky low DO situation generally appears under a mild flow condition with a low Reynolds number, and thus, this technique may be practically introduced.

\section{B. Aims of the present study}

The intensity of the turbulence and its space-time scales in a free-surface layer are significantly influenced by the bottom roughness and slope. Therefore, local bottom conditions are expected to control the gas transfer beneath a free surface. To reveal the response properties of the gas transfer velocity to local variations in the mean flow and turbulence, we do not need to use the whole tank method; rather, it suffices to use other methods such as the laser-induced fluorescence (LIF) technique, which can locally measure the gas transfer velocity. However, it is difficult to conduct the LIF technique in a $10-\mathrm{m}$ long circulating flume due to difficulties related to the dissolution of the fluorescence dye with a constant concentration in space and the setup of a laser light source projecting homogeneously over a large-scale measurement plane.

To address this problem, the present study measured the streamwise profile of the thickness of the DO concentration layer by traversing an extra-fine needle-type DO probe through a portion of the flow. The results are then used to evaluate the distribution of the gas transfer velocity. Laboratory experiments were performed in a straight open channel with a suitably shaped wedge to locally accelerate the streamwise current. In addition, this study introduces a 
theoretical equation of the distribution of the concentration boundary layer thickness, which describes how the formation of the concentration boundary layer thickness is governed by the streamwise gradient of the free-surface mean velocity and the strength of the turbulent diffusivity near the free surface. The present study discusses the influences of the mean flow acceleration and the turbulent diffusivity on the local gas transport in open-channel flows using both experimental and theoretical work. Many prior studies proposed and examined physical modeling of bulk-scale gas transfer. However, there is no information on the local gas transfer in non-uniform flow fields. To provide a breakthrough regarding this difficult task, this study suggested a mathematical model to explain the local gas transfer in the accelerated open-channel zone. Additionally, the physical mechanism was investigated by comparison with experiments of the free-surface velocity and the CBT profiles.

Section II describes the theoretical work used to consider the streamwise variation in the concentration boundary layer thickness. Section III describes the experimental procedures for the measurements of the boundary layer thickness and the velocity components in the laboratory flume. In Sec. IV, the hydrodynamic characteristics and gas transfer are considered based on the measurement results under the locally accelerated condition. A comparison with the newly proposed theory reveals the detailed mechanisms of the developed boundary layer thickness related to the gas transfer in open-channel flows. In Sec. V, our conclusions are described based on the results.

\section{THEORETICAL EXPANSION OF THE CONCENTRATION BOUNDARY LAYER THICKNESS}

The present study considers the spatial development of the gas concentration boundary layer thickness near a free surface based on a two-dimensional (2D) coordinate system, as shown in Fig. 1. $x$ and $y$ are the streamwise and downward-vertical coordinates with their origins at the flume inlet and the free surface, respectively, $y^{\prime}$ is the upward-vertical coordinate with its origin at the flume bottom, $U$ and $V$ are the time-averaged velocity components corresponding to the $x$ and $y$ directions, respectively, and $H$ is the water depth.

The local gas flux beneath the air-water interface is significantly related to the CBT. The concentration profile of the dissolved gas is assumed to fit the following exponential function in the two-dimensional field:

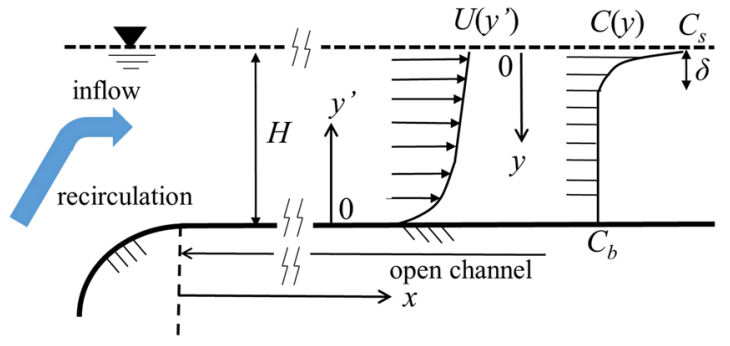

FIG. 1. 2D coordinate system for analysis of the concentration boundary layer thickness.

$$
\frac{C-C_{b}}{C_{s}-C_{b}}=e^{-\frac{y}{\delta}},
$$

where subscripts $b$ and $s$ indicate the bulk layer and the free surface, respectively; $C=C(x, y)$ is the time-mean gas concentration in the stationary stage, which depends on the vertical position; $C_{s}$ is the saturated concentration; $C_{b}$ is the bulk concentration; and $\delta(x)$ is a parameter that determines the time-mean concentration profile, which is defined as the CBT here. Equation (1) was previously validated by Herlina and Jirka.

Fick's law tells us that the gas flux is controlled by the vertical gradient of the gas concentration formed very near the free surface. The combination of this fact and Eq. (1) allows the gas flux to be expressed using the CBT. The influence of the bottom-oriented turbulence approaches the free surface for larger Re conditions, and this results in a smaller $\delta$ and a larger gas flux. However, this mechanism has not been observed because the accurate measurement of $\delta$ is very difficult. In particular, few studies have examined the formation of CBT in open-channel turbulence. Accordingly, this section conducts a theoretical study of the CBT in 2D open-channel flow prior to the experimental work. This study assumes a mild free surface, i.e., one without time-dependent free-surface variations.

The instantaneous streamwise, vertical, and spanwise velocities and concentration can be decomposed into their mean and fluctuation components, which are expressed as

$$
\begin{gathered}
\tilde{u}=U+u, \\
\tilde{v}=V+v, \\
\tilde{w}=W+w, \\
\tilde{c}=C+c,
\end{gathered}
$$

where the tilde, capital letters, and small letters indicate the instantaneous, time-averaged, and fluctuation components, respectively. These mean and fluctuation variables depend on the coordinates $(x, y, z)$.

The $3 \mathrm{D}$ transport equation of the dissolved gas concentration is expressed as

$$
\frac{\partial \tilde{c}}{\partial t}+\frac{\partial \tilde{c} \tilde{u}}{\partial x}+\frac{\partial \tilde{c} \tilde{v}}{\partial y}+\frac{\partial \tilde{c} \tilde{w}}{\partial z}=D\left(\frac{\partial^{2} \tilde{c}}{\partial x^{2}}+\frac{\partial^{2} \tilde{c}}{\partial y^{2}}+\frac{\partial^{2} \tilde{c}}{\partial z^{2}}\right),
$$

where $D$ is the molecular diffusion coefficient.

The Reynolds-averaged form ${ }^{30}$ of Eq. (6) can be written as

$$
\begin{aligned}
\frac{\partial C U}{\partial x} & +\frac{\partial C V}{\partial y}+\frac{\partial C W}{\partial z} \\
& =D\left(\frac{\partial^{2} C}{\partial x^{2}}+\frac{\partial^{2} C}{\partial y^{2}}+\frac{\partial^{2} C}{\partial z^{2}}\right)-\frac{\partial \overline{c u}}{\partial x}-\frac{\partial \overline{c v}}{\partial y}-\frac{\partial \overline{c w}}{\partial z},
\end{aligned}
$$

where the overbar indicates the time-averaged operator. The time variation in $C$ is very small in the present gas transfer situation, and the unsteady term is not considered in Eq. (7).

Assuming that spanwise transport is negligible and the concentration flux is much more significant in the vertical direction than the horizontal ones, the $2 \mathrm{D}$ transport equation of the dissolved gas concentration can be obtained as

$$
\frac{\partial C U}{\partial x}+\frac{\partial C V}{\partial y}=D\left(\frac{\partial^{2} C}{\partial x^{2}}+\frac{\partial^{2} C}{\partial y^{2}}\right)-\frac{\partial \overline{c u}}{\partial x}-\frac{\partial \overline{c v}}{\partial y} .
$$


The incompressible 2D continuity equation is as follows when the water density is assumed to be constant:

$$
\frac{\partial U}{\partial x}+\frac{\partial V}{\partial y}=0
$$

Hence, Eq. (8) is transformed into

$$
U \frac{\partial C}{\partial x}+V \frac{\partial C}{\partial y} \approx D \frac{\partial^{2} C}{\partial y^{2}}-\frac{\partial \overline{c v}}{\partial y} .
$$

This study defined the free-surface velocity as $U_{s}=U(y=0)$ $=U\left(y^{\prime}=H\right)$. The following theoretical expansion assumes that $U$ $\approx U_{s}$ for $0 \leq y \leq \delta$ and also $\partial U / \partial x \approx \partial U_{s} / \partial x$ for $0 \leq y \leq \delta$ in the concentration boundary layer. The integral of Eq. (10) over $y=0$ to $\delta$ results in the streamwise variation in $\delta$.

The integral of Eq. (9) from the free surface $(y=0)$ to an arbitrary depth very near the free $\operatorname{surface}\left(y=y_{i}\right)$ is

$$
\begin{aligned}
\int_{0}^{y_{i}} \frac{\partial U}{\partial x} d y & =-\int_{0}^{y_{i}} \frac{\partial V}{\partial y} d y=-\left[V\left(y_{i}\right)-V(0)\right]=-V\left(y_{i}\right) \\
& \leftrightarrow V(y)=-\int_{0}^{y} \frac{\partial U}{\partial x} d y .
\end{aligned}
$$

Using Eq. (11), the integral of the second term on the left-hand side of Eq. (10) can be expressed [see (A1)] as

$$
\int_{0}^{\delta} V \frac{\partial C}{\partial y} d y=-C(\delta) \frac{\partial U_{s}}{\partial x} \delta+\int_{0}^{\delta} C \frac{\partial U}{\partial x} d y .
$$

Therefore, we can write the integral of the left-hand side of Eq. (10) as

$$
\begin{aligned}
\int_{0}^{\delta} & \left(U \frac{\partial C}{\partial x}+V \frac{\partial C}{\partial y}\right) d y \\
& =\int_{0}^{\delta} U \frac{\partial C}{\partial x} d y+\int_{0}^{\delta} C \frac{\partial U}{\partial x} d y-C(\delta) \frac{\partial U_{s}}{\partial x} \delta .
\end{aligned}
$$

The gas concentration $C(y)$ varies in the depth direction. Therefore, a preparatory calculation is carried out to explore Eq. (13).

Equation (1) can be replaced by the following equation using the concentration difference $\Delta C=C_{s}-C_{b}$ :

$$
C=e^{-\frac{y}{\delta}} \Delta C+C_{b}
$$

The concentration at the depth of the CBT $(y=\delta)$ is

$$
C(\delta)=\frac{\Delta C}{e}+C_{b}
$$

Differentiating Eq. (14) results in

$$
\frac{\partial C}{\partial y}=-\frac{\Delta C}{\delta} e^{-\frac{y}{\delta}}
$$

Therefore, the concentration gradients at $y=0$ and $\delta$ are as follows:

$$
\left.\frac{\partial C}{\partial y}\right|_{y=0}=-\frac{\Delta C}{\delta},\left.\quad \frac{\partial \mathrm{C}}{\partial \mathrm{y}}\right|_{y=\delta}=-\frac{\Delta C}{\delta e} .
$$

Furthermore, the integration of Eq. (14) from $y=0$ to $\delta$ is as follows [see (A2)]:

$$
\int_{0}^{\delta} C d y=\delta\left(-\frac{\Delta C}{e}+\Delta C+C_{b}\right)
$$

This study explored Eq. (13) using Eqs. (14)-(18). First, the first term on the right-side hand of Eq. (13) can be calculated in the following manner [see (A3)]:

$$
\int_{0}^{\delta} U \frac{\partial C}{\partial x} d y \approx U_{s} \int_{0}^{\delta} \frac{\partial C}{\partial x} d y=U_{s}\left\{\Delta C\left(1-\frac{1}{e}\right)+C_{b}\right\} \frac{\partial \delta}{\partial x} .
$$

Second, the following calculation was conducted to integrate the second term on the right-hand side of Eq. (13) [see (A4)]:

$$
\int_{0}^{\delta} y e^{-\frac{y}{\delta}} d y=-\frac{2 \delta^{2}}{e}+\delta^{2}=\delta^{2}\left(1-\frac{2}{e}\right)
$$

The second term on the right-hand side of Eq. (13) can be integrated using Eq. (20) [see (A5)] as follows:

$$
\int_{0}^{\delta} C \frac{\partial U}{\partial x} d y \approx \frac{\partial U_{s}}{\partial x} \delta\left\{\Delta C\left(1-\frac{1}{e}\right)+C_{b}\right\} .
$$

Therefore, we can integrate the left-hand side of Eq. (10) in the following manner using Eqs. (13), (14), (19), and (21) [see (A6)]:

$$
\begin{aligned}
\int_{0}^{\delta} & \left(U \frac{\partial C}{\partial x}+V \frac{\partial C}{\partial y}\right) d y \\
& =U_{s}\left\{\Delta C\left(1-\frac{1}{e}\right)+C_{b}\right\} \frac{\partial \delta}{\partial x}+\frac{\partial U_{s}}{\partial x}\left(1-\frac{2}{e}\right) \Delta C \delta .
\end{aligned}
$$

Conversely, the first term on the right-hand side of Eq. (10) can be integrated in the following manner:

$$
\begin{aligned}
\int_{0}^{\delta} D \frac{\partial^{2} C}{\partial y^{2}} d y & =D \int_{0}^{\delta} \frac{\partial^{2} C}{\partial y^{2}} d y \\
& =D\left[\frac{\partial C}{\partial y}\right]_{0}^{\delta}=D\left(-\frac{\Delta C}{\delta e}+\frac{\Delta C}{\delta}\right)=\frac{D \Delta C}{\delta}\left(-\frac{1}{e}+1\right)
\end{aligned}
$$

Introducing the gradient diffusion hypothesis, ${ }^{31}$ the concentrationvertical velocity correlation can be expressed as

$$
-\overline{c v}=D_{t} \frac{\partial C}{\partial y}
$$

where $D_{t}$ is a turbulent diffusion coefficient. The gradient diffusion hypothesis expresses the local scalar flux at a point, and the influences of the flow situation and turbulence strength are aggregated in $D_{t}$. This variable depends on the vertical depth at a certain streamwise position. Thus, this hypothesis is expected to be used in the accelerated flow and in the uniform flow. Hamba ${ }^{32}$ examined this hypothesis of scalar flux in the channel turbulence by using DNS. He showed that the largest error occurs near the wall where the scalar gradient is large. Regardless, it is not more than a $10 \%$ order shift compared with the DNS result. Thus, the gradient diffusion hypothesis is expected to be used in the present theoretical expansion without serious difficulties. 
Therefore, the integral of the second term on the right-hand side of Eq. (10) could be calculated [see (A7)] as

$$
\int_{0}^{\delta}-\frac{\partial \overline{c v}}{\partial y} d y=-\frac{\Delta C}{\delta e} D_{t}(\delta)
$$

where $D_{t}(0)=0$.

Using Eqs. (23) and (25), the integral of the right-hand side of Eq. (10) could be obtained as follows:

$$
\int_{0}^{\delta}\left(D \frac{\partial^{2} C}{\partial y^{2}}-\frac{\partial \overline{c v}}{\partial y}\right) d y=\frac{\Delta C}{\delta}\left\{D\left(1-\frac{1}{e}\right)-\frac{D_{t}(\delta)}{e}\right\} .
$$

Finally, this resulted in the streamwise variation in CBT, as expressed by

$$
\begin{aligned}
U_{s}\{\Delta & \left.C\left(1-\frac{1}{e}\right)+C_{b}\right\} \frac{\partial \delta}{\partial x}+\frac{\partial U_{s}}{\partial x}\left(1-\frac{2}{e}\right) \Delta C \delta \\
= & \Delta C\left\{\left(1-\frac{1}{e}\right) D-\frac{1}{e} D_{t}(\delta)\right\} \delta^{-1} \\
& \leftrightarrow U_{s}\left(1-\frac{1}{e}+\frac{C_{b}}{\Delta C}\right) \frac{\partial \delta}{\partial x}+\frac{\partial U_{s}}{\partial x}\left(1-\frac{2}{e}\right) \delta \\
= & \left\{\left(1-\frac{1}{e}\right) D-\frac{1}{e} D_{t}(\delta)\right\} \delta^{-1} \\
& \leftrightarrow \frac{\partial \delta}{\partial x}+\frac{\partial U_{s}}{\partial x}\left(1-\frac{2}{e}\right) \frac{1}{C_{o} U_{s}} \delta \\
= & \frac{1}{C_{o} U_{s}}\left\{\left(1-\frac{1}{e}\right) D-\frac{1}{e} D_{t}(\delta)\right\} \delta^{-1} \\
\leftrightarrow & \frac{\partial \delta}{\partial x}=A \delta+B \delta^{-1},
\end{aligned}
$$

where

$$
\begin{gathered}
A=-\frac{\partial U_{s}}{\partial x}\left(1-\frac{2}{e}\right) \frac{1}{C_{o} U_{s}}, \\
B=\frac{1}{C_{o} U_{s}}\left\{\left(1-\frac{1}{e}\right) D-\frac{1}{e} D_{t}(\delta)\right\}, \\
C_{o}=1-\frac{1}{e}+\frac{C_{b}}{\Delta C} .
\end{gathered}
$$

Equation (27) is the first-order Bernoulli differential equation, and its solution is

$$
\delta(x)=\left[e^{-2 \int_{0}^{x} A d X}\left\{\int_{0}^{x} 2 B e^{2 \int_{0}^{x} A d X} d X+C_{1}\right\}\right]^{\frac{1}{2}} .
$$

Here, $C_{1}$ is an integral constant. When the flow is uniform in the streamwise direction, Eq. (31) can be further simplified as

$$
\delta(x)=\left(\int_{0}^{x} 2 B d X+C_{1}\right)^{\frac{1}{2}} .
$$

Sections III and IV consider the developmental properties of CBT by comparing this theory with the measurement results. flux $F$,

The gas transfer velocity $k_{L}$ can be defined using the gas transfer

$$
F \equiv k_{L} \Delta \mathrm{C} .
$$

Conversely, the following form can be obtained using Fick's law: ${ }^{33}$

$$
F \equiv-D \frac{\partial C}{\partial y}(y=0)
$$

These two forms lead to

$$
k_{L}(x)=\frac{D}{\delta(x)} .
$$

This is equivalent to the film model proposed by Lewis and Whitman. ${ }^{34}$ The molecular diffusivity is constant without temperature change. Therefore, when we measure the streamwise profile of CBT $\delta$, the gas transfer velocity $k_{L}$ can be evaluated using Eq. (35).

\section{EXPERIMENTAL SETUP}

\section{A. The recirculating flume system and the hydraulic conditions}

Figure 2 shows the recirculating laboratory flume, which was $40 \mathrm{~cm}$ in width, $50 \mathrm{~cm}$ in height, and $16 \mathrm{~m}$ in length. Two types of experiments, i.e., velocity measurements using the PIV technique and CBT measurements using the extra-fine needle-type DO probe, were conducted while varying the fetch (the distance from the channel inlet). This study placed a trapezoidally shaped wedge in the section from $7.5 \mathrm{~m}$ to $11.5 \mathrm{~m}$ from the channel inlet. The longitudinal length of the acceleration zone $L_{s}$ was $75 \mathrm{~cm}$, and the channel bottom was raised by $3 \mathrm{~cm}$. This resulted in the cross-sectional area being reduced by half, as shown in Fig. 2. Twelve test sections were chosen around the upstream-side wedge where the streamwise current was expected to gradually accelerate. The origin of the $x$-axis was placed at the leading edge of the acceleration zone. The hydraulic conditions were as follows: $U_{m}=10 \mathrm{~cm} / \mathrm{s}$ and $H=6 \mathrm{~cm}$ were the bulk mean velocity and the water depth in the upstream flat-bottom zone, respectively. $\mathrm{Re}=6000$ was the Reynolds number based on $U_{m}$ and $H$, and $\mathrm{Fr}=0.13$ was the Froude number, which implies a flat free surface. The room and water temperatures were maintained at $25^{\circ} \mathrm{C}$ using air conditioners.

\section{B. DO measurements with a fine probe}

A concentration profile of the dissolved gas very near the free surface is required to evaluate the CBT. Herlina and Jirka ${ }^{8}$ measured the DO profile reasonably well using LIF. The advantage of LIF is that it measures the instantaneous concentration boundary layer and can be used to evaluate the local gas flux via the concentrationvelocity correlation. However, applying LIF in a laboratory flume on the order of $10 \mathrm{~m}$ in length is very difficult due to the difficulty of achieving a uniform dissolution of the fluorescent material. Therefore, this study uses an optical fine DO probe (PreSens, Microx4), as shown in Fig. 3. An optical type of probe is advantageous for accurate measurements even in very low-speed currents, for which classical electrode-type probes cannot be used. The probe was a 0.8$\mathrm{mm}$ thick fine needle, which was used over a miniscule volume. However, the tip size of the probe is still comparable with the typical CBT of DO, which is generally less than $1 \mathrm{~mm} .{ }^{33}$ Hence, this study measured concentration profiles ten times at each fetch to examine the repeatability of this probe. An additional optical DO probe (YSI, ProODO) was situated at the mid-depth near the outlet 


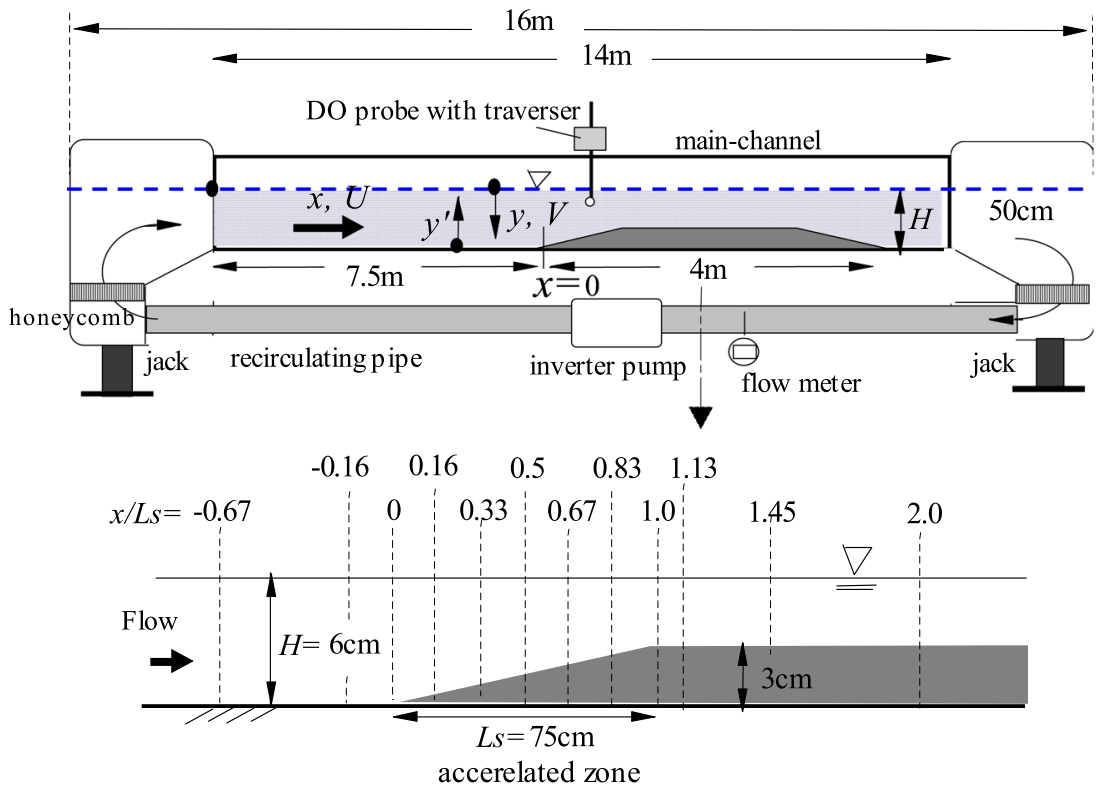

FIG. 2. Experimental flume in which a suitably shaped wedge is situated to accelerate the main stream. of the flume to monitor the time variation in the DO in the bulk layer once every minute. Surface cleanliness affects significantly the nearsurface turbulence and gas transfer (e.g., Refs. 35 and 36). Before the measurement, water was circulated within the flume for $10 \mathrm{~h}$ for temperature adjustment. Through this procedure, floating dusts and fine substances in the water current were removed by using a net of fine meshes placed downstream of the flume.

The CBT measurements were composed of several steps after surface-cleaning work. The main channel was deaerated prior to the measurement step by dissolving a certain amount of sodium sulfite. Figure 4(a) shows the time variation in the DO in the bulk layer; $C_{b}(t)$ is normalized by an initial value $C_{b}(0)$ and a saturated value $C_{s}$. This value is nearly constant and close to zero in the initial stage. After the full consumption of the deaeration chemical, the DO returns to its previous level with time. Figure 4(b) shows the variation in the logarithm of the normalized $C_{b}(t)$ corresponding to Fig. 4(a). The reaeration coefficient $k_{2}=k_{L} / H$ can be evaluated via the slope in the linear reduction stage. This is called the whole tank method. Even though the $k_{2}$ or $k_{L}$ value obtained using this method is not a local value but rather a global value of the entire recirculating

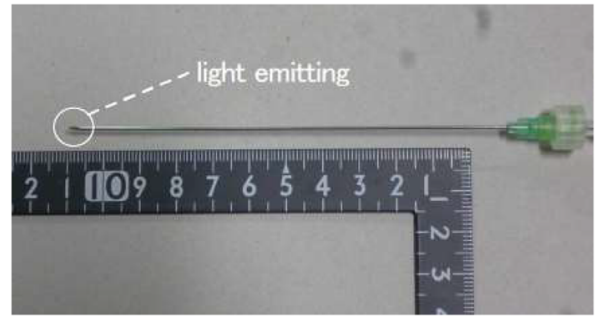

FIG. 3. Optical fine DO probe with a $0.8 \mathrm{~mm}$ thick fine needle. flume system, $k_{L}$ can be constant, irrespective of the time variation in $C_{b}(t)$ in the linear reduction stage.

Therefore, the measurement of the DO profile was conducted in the linear reduction stage. Preliminary tests specified the beginning and end timings of the linear reduction stage in advance. The vertical traversing of the fine DO probe with $0.01-\mathrm{mm}$ accuracy obtained the mean concentration at ten depths, including the surface layer at $y=0.3 \mathrm{~mm}$ and the bulk layer at $y=10 \mathrm{~mm}$. The sampling duration was $30 \mathrm{~s}$ every $2 \mathrm{~s}$ at each depth. When the DO probe was moved to another depth, the sampling resumed after waiting $40 \mathrm{~s}$ to take the stability of the signal input into account. The fetches of the test sections were chosen at $x / L_{s}=-0.67,-0.16,0,0.16,0.33$, $0.5,0.67,0.83,1.0,1.13,1.45$, and 2.0 . Figure 5 shows an example of the normalized DO profile, $\left(C-C_{b}\right) / \Delta C$ in the vertical direction obtained in the accelerated zone of $x / L_{s}=0.67$ and 2.0 with error bars. Fitting Eq. (1) using a least-squares method, a suitable value of $\delta$ was obtained for each streamwise position. We are sure that most measured points are out of the CBT. Hence, the reliability of extrapolation is very important. The standard deviation reduces apart from the free surface, and therefore, the extrapolation using several points with a small deviation is expected to be sufficient to evaluate quantitatively the CBT.

\section{Current velocity measurements with PIV}

PIV is a useful technique to non-intrusively obtain the turbulent velocity field. Our PIV system used a laser light sheet (LLS), which was generated by a $2-W$ YAG continuous laser source. The present study set up two types of laser-illuminated planes. One was a horizontally illuminated PIV (H-PIV) in which a time series of horizontal images on the $x-z$ plane very near the free surface were acquired to obtain information concerning the near free-surface mean velocity and related turbulence, including the surface velocity divergence. The $2-\mathrm{mm}$ thick LLS was horizontally projected just 
(a)

$\left(C_{b}(t)-C_{b}(0)\right) /\left(C_{s}-C_{b}(0)\right)$

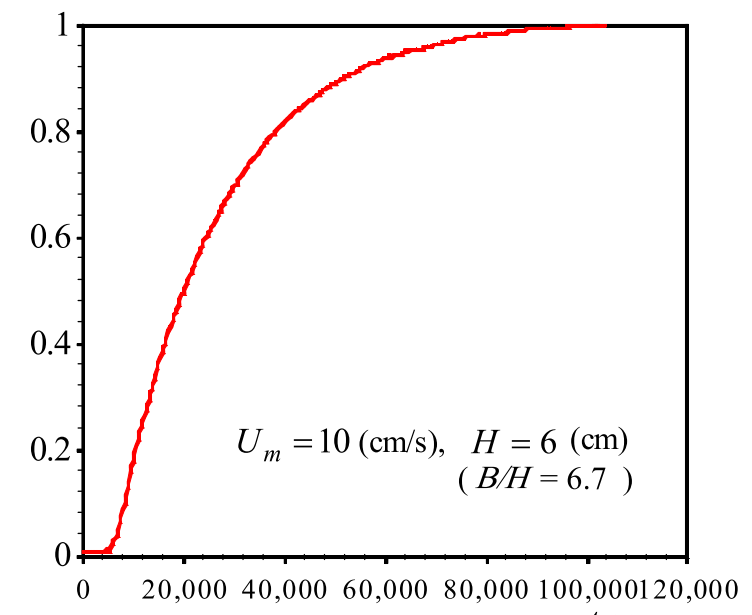

$t(\mathrm{~s})$

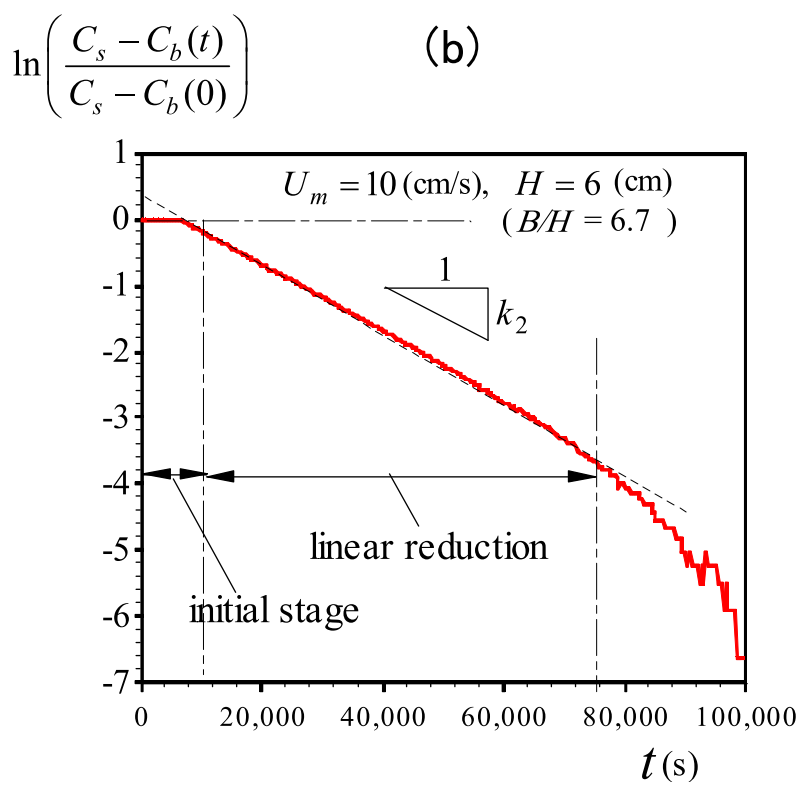

FIG. 4. Time variation in the DO in the bulk layer, $C_{b}(t)$ normalized by an initial value $C_{b}(0)$ and a saturated value $C_{s}$. (a) Linear plot, (b) semi-logarithmic plot.

$1 \mathrm{~mm}$ below the interface because clear images could not be acquired at the interface elevation. ${ }^{26}$ The covering image area on the center line was $10 \times 10 \mathrm{~cm}^{2}$ with an interrogation window size of 1.2 $\times 1.2 \mathrm{~mm}^{2}$.

The time-averaged streamwise velocity, $U_{s}(x)$, turbulence kinetic energy in the free surface, $k_{s}(x) \equiv\left(u_{s}^{\prime}(x)^{2}+w_{s}^{\prime}(x)^{2}\right) / 2$, and divergence intensity, $\beta^{\prime}(x) \equiv \sqrt{\overline{\beta^{2}}}$, were used at the center of the horizontal image obtained at each fetch in which $u_{s}^{\prime} \equiv \sqrt{\overline{u_{s}^{2}}}$
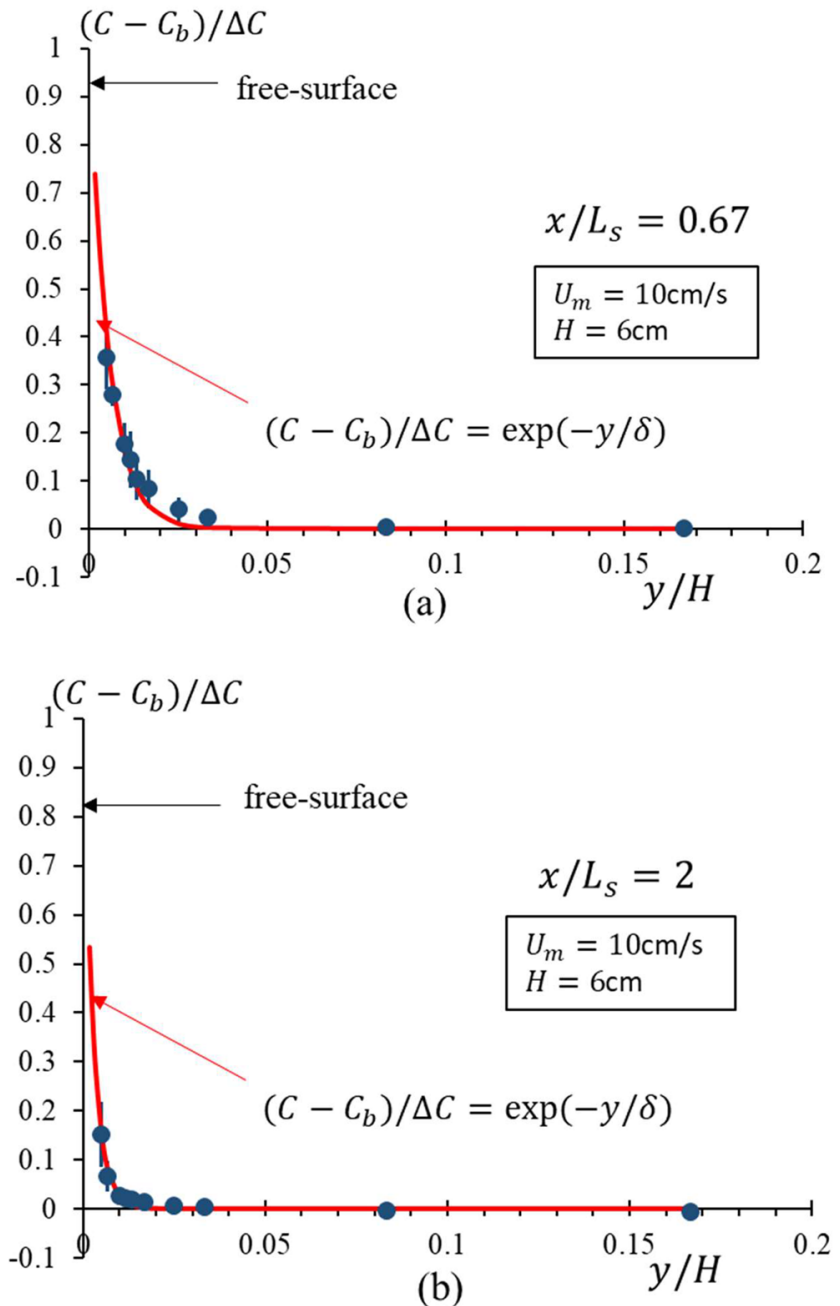

FIG. 5. Vertical profile of $D O$ in the linear reduction stage, $\left(C-C_{b}(t)\right) / \Delta C$.

and $w_{s}^{\prime} \equiv \sqrt{\overline{w_{s}^{2}}}$, which are turbulence intensities in the free surface for streamwise and spanwise directions, respectively. $\beta(x)$ $\equiv-\left(\partial u_{s} / \partial x+\partial w_{s} / \partial z\right)=\partial v_{s} / \partial y$ is the fluctuation of velocity divergence in the free surface. The occurrences of instantaneous upward and downward currents under the free surface induce the negative and positive signs, respectively. The divergence and turbulence intensities mean root mean square (RSM) values of instantaneous divergence and turbulence components, respectively.

The specific density and mean diameter of the tracer particles were $1.02 \mu \mathrm{m}$ and $100 \mu \mathrm{m}$, respectively. The spatial and temporal distributions of the tracer patterns were determined using a highspeed CMOS camera situated over the free surface. The frame rate was $500 \mathrm{~Hz}$, depending on the bulk mean velocity. The frame rate corresponds to the time lag between two consecutive images and is used to calculate the $2 \mathrm{D}$ velocity components. The sample rate, which specifies the time interval between the obtained velocity data, was given as $30 \mathrm{~Hz}$ by the function generator. ${ }^{37}$ The repeatability standard deviation was evaluated for the mean streamwise velocity 
and Reynolds stress in the same manner as done by Sanjou and Nezu. ${ }^{38}$ They were $\sigma_{U} / U=0.05$ and $\sigma_{u w} /|\overline{u w}|=0.27$, respectively. The positions of the high-speed camera varied with the streamwise direction in the same manner as in the above-mentioned DO experiment. The DO and PIV experiments were performed independently, and the obtained velocity components at the image center were used for the following analysis.

\section{RESULTS AND DISCUSSION}

\section{A. Streamwise variations in the mean velocity and turbulence on the free surface}

Many researchers have focused on the contributions of the velocity acceleration to the promotion of turbulence. Narayanan and Ramjee ${ }^{39}$ obtained an accelerated turbulent boundary layer in a wind tunnel with a suitably designed wedge. They provided the mean velocity profile and turbulent statistics and obtained universal properties via a proper scaling without a Reynolds number dependency. Ranjan and Narasimha ${ }^{40}$ reviewed several significant experiments, providing detailed measurement results. Experimental results by Narayanan and Ramjee ${ }^{39}$ (labeled N-R in the legends of Figs. 6, 7, and 11), Blackwelder and Kovasznay ${ }^{41}$ (B-K), Warnack and Fernholz ${ }^{42}$ (W-F), Escudier et al. ${ }^{43}$ (Es), and Bourassa and Thomas ${ }^{44}(\mathrm{~B}-\mathrm{T})$ are also plotted for comparison, where the free-stream velocity $U_{\infty}$ is used as the characteristic velocity scale.

Figure 6 shows the streamwise variations in the mean surface velocity normalized by the entrance velocity, $U_{s} / U_{s}(x=0)$. The present results were obtained from the PIV measurements. $x / L_{s}$ $=0$ and 1 indicate the entrance and exit positions of the acceleration zone, respectively. The following common tendencies of these experiments were observed. In the acceleration zone, the mean velocity

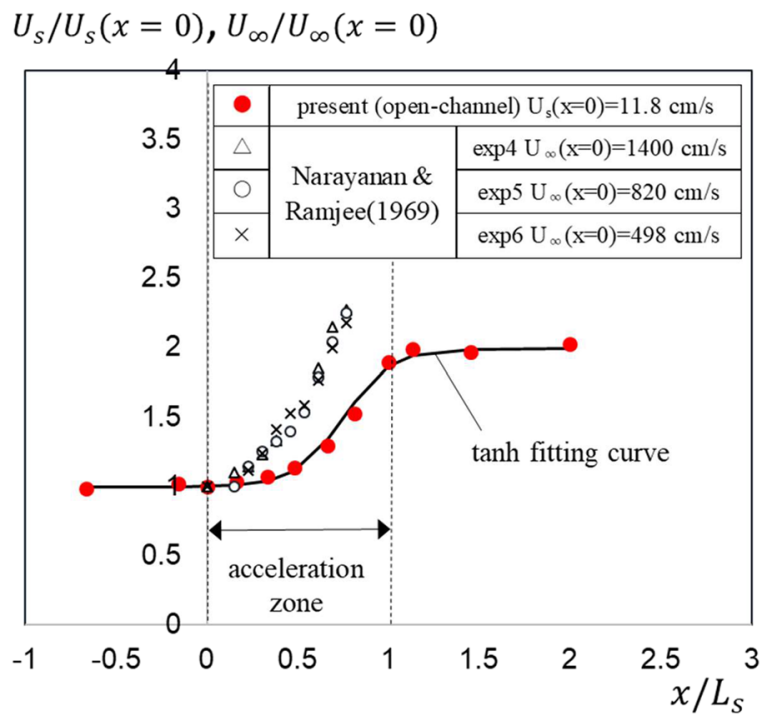

FIG. 6. Streamwise variations in mean surface velocity normalized by the entrance velocity, $U_{S} / U_{S}(x=0)$. has a tangent-hyperbolic-like curve. The velocity gradient is more remarkable in the downstream side of the acceleration zone $(0.5$ $\left.<x / L_{s}<1\right)$ than in the upstream-side $\left(0<x / L_{s}<0.5\right)$, which is because the bottom wedge more significantly influences the freesurface current in the shallower situation. The free-surface velocity continued to increase a bit downstream from the exit due to the redevelopment of the boundary layer.

The measured profile fits well with the following hyperbolic tangent function:

$$
U_{s}(x)=\frac{U_{s 1}+U_{s 2}}{2}-\frac{U_{s 1}-U_{s 2}}{2} \tanh \left(A_{U}\left(x-x_{U}\right)\right),
$$

where $U_{s 1}=12.2 \mathrm{~cm} / \mathrm{s}, U_{s 2}=24.0 \mathrm{~cm} / \mathrm{s}, A_{U}=0.05$, and $x_{U}$ $=60 \mathrm{~cm}$. The fitting function was found to be shifted downstream in the acceleration zone, which means that it still increases at the exit position $\left(x / L_{s}=1\right)$ corresponding to the streamwise shift of the profile, $U_{s}(x)$. The formation of the velocity boundary layer restarts to develop over the shallow flat zone $\left(x / L_{s}=1\right)$, and thus, the reduction in the bottom velocity due to the bottom friction lets the free surface velocity continue to accelerate a bit downstream of the exit position $\left(x / L_{s}=1\right)$.

The acceleration level is generally evaluated using the acceleration parameter, $K(x)$, as defined by Morretti and Kays,

$$
K \equiv v / U_{\infty}^{2} \frac{d U_{\infty}}{d x} \sim v / U_{s}^{2} \frac{d U_{s}}{d x} .
$$

The present study evaluated $K$ by replacing $U_{\infty}$ with $U_{s}$. Figure 7 shows the streamwise variation in the acceleration parameter. All of the results suggest that $K$ varies significantly in the acceleration zone. Here, the maximum value of $K$, which is $8.8 \times 10^{-6}$ in the present study, is larger than the values found in the previous wind experiments. The criteria of the onset values of the relaminarization proposed by Narayanan and Ramjee, ${ }^{39}$ Kline et al., ${ }^{46}$ and Blackwelder and Kovasznay ${ }^{41}$ range from $3.0 \times 10^{-6}$ to $3.6 \times 10^{-6}$. Therefore, the present experiment is expected to experience the relaminarization process in the open-channel flow, as explained later. These

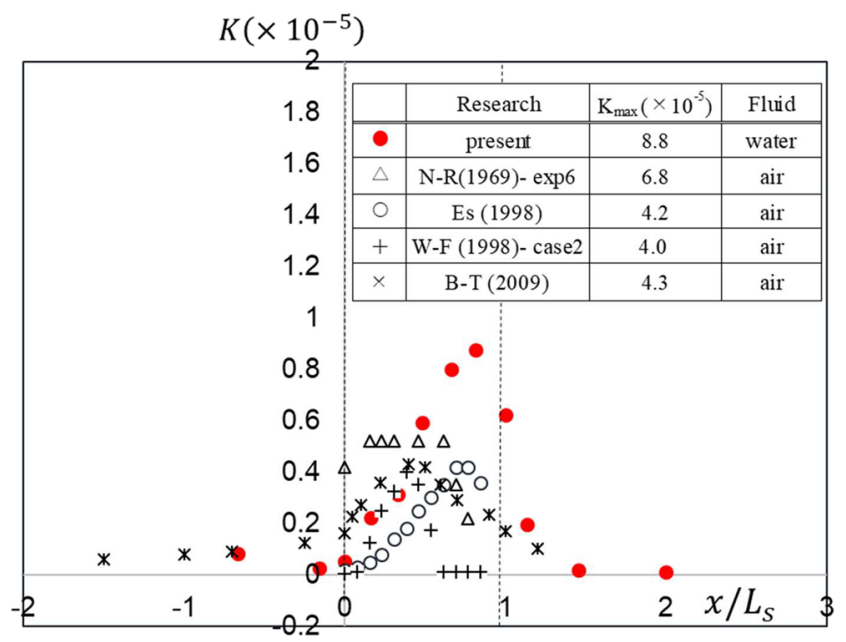

FIG. 7. Streamwise variation in the pressure gradient parameter $K$. 


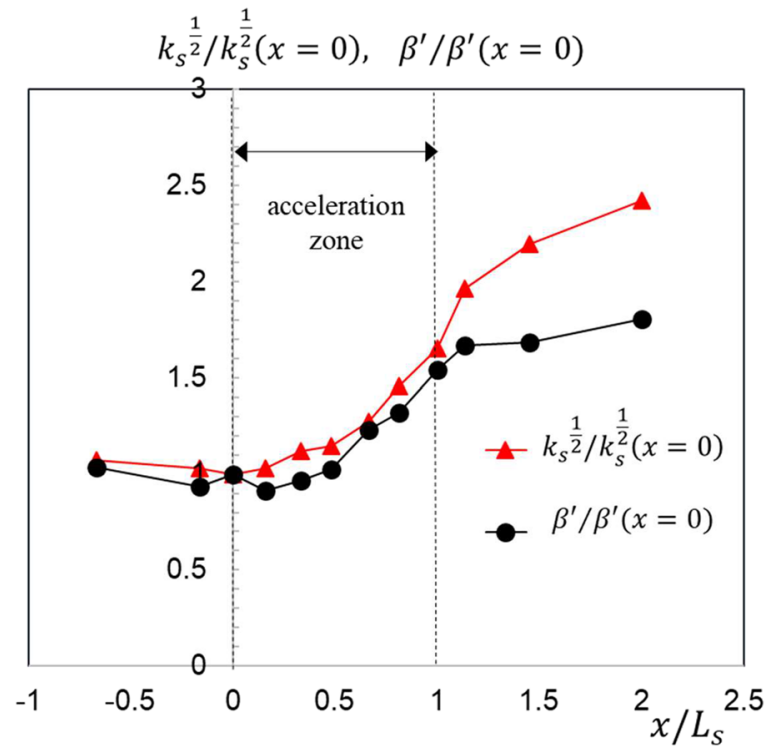

FIG. 8. Streamwise variations in turbulence kinetic energy at the free-surface $(y=0) k_{s}$ and surface divergence intensity $\beta^{\prime}$

results imply that an accelerated flow has common developmental features that are irrespective of the fluid type. The maximum peak of $K$ shifts a bit downstream in the same fashion as in the work of Escudier et al. ${ }^{43}$

Figure 8 shows the streamwise variations in the turbulent kinetic energy at the free surface $(y=0) k_{s}$ and the SD intensity $\beta^{\prime}$. Common features can be observed for the normalized $k_{s} / k_{s}(x=0)$ and $\beta^{\prime} / \beta^{\prime}(x=0)$. These values are nearly constant prior to the beginning of the acceleration zone, and they start to increase due to the channel reduction in the acceleration zone. The streamwise variations in $k_{s}$ and $\beta^{\prime}$ are small in the upstream side in the acceleration zone, corresponding to the variation in the free-surface velocity. It is notable for them to start increasing not at the entrance $x / L_{s}=0$, but at $x / L_{s}=0.33$. This may also be because it takes a certain time until the bottom-oriented turbulence arrives at the free surface. Furthermore, it is of particular significance that they still increase at the exit. These tendencies imply the relaminarization observed in the accelerated flow. That is to say, the reduced turbulence generation in the accelerated zone starts to restore there. This topic is considered in detail later. The free-surface flow is accelerated in the transition at the leading edge of a submerged vegetation canopy. A strong upward current out of the canopy appears, and the acceleration and deceleration of the mean streamwise flows are promoted in the initial adjustment zone. The formation of a depth-scale mixing layer is completed downstream of the initial adjustment (e.g., Ref. 47). The free-surface turbulence at the leading edge of the acceleration zone is also expected to develop later than the mean streamwise velocity in the same manner as in the initial adjustment of a submerged vegetation patch.

Positive and negative divergences are known to appear in space and time in open-channel flows. ${ }^{26}$ They refer to the vertical velocity fluctuation, which is directly related to downward and upward gas transports. Thus, the gas transfer rate is expected from Fig. 8 to increase with the flow acceleration. Herlina and Wissink ${ }^{48}$ asserted that local surface-attached vortex structures (SAVS) having the surface-normal axis are stretched and strengthened. In the smallscale converging zone accompanied with downwelling currents, gas transfer is expected to be related to the interactions between the SAVS that promote the horizontal mixing and the longitudinal secondary vortex that promotes the vertical mixing.

\section{B. Streamwise variation in the CBT}

Figure 9 shows the streamwise variation in $\delta$, which is measured by the extra-fine DO probe in which the error bars that are based on the standard deviation are included. The normalized repeatability standard deviation, $\sigma_{\delta} / \delta$, ranged from 0.18 to 0.4 . The gas transfer rate and the related CBT are sensitive to the air and water temperatures. Although the temperatures were kept constant by the air conditioner as mentioned above, not the present one-way control but the advanced management, such as via feedback computer control, will reduce measurement errors. A significant reduction in $\delta$ can be observed in the acceleration zone. In particular, $\delta$ starts to decrease around $x / L_{s}=0.33$, corresponding to the variation in the free-surface turbulence. This fact implies that the gas transfer flux is closely related to the formation of the free-surface turbulence. In addition, the CBT downstream of the acceleration zone is half its value at the beginning of the acceleration. Note that the CBT is controlled by two factors: the mean flow acceleration and the development of the free-surface turbulence.

It was found that decreasing the CBT tendency can be reasonably explained despite the $10 \%$ order standard deviations. Thus, this study concluded that the present $0.8-\mathrm{mm}$-diameter DO probe has sufficient repeatability and reliability to study the streamwise variation in the CBT in non-uniform flows, such as the accelerated

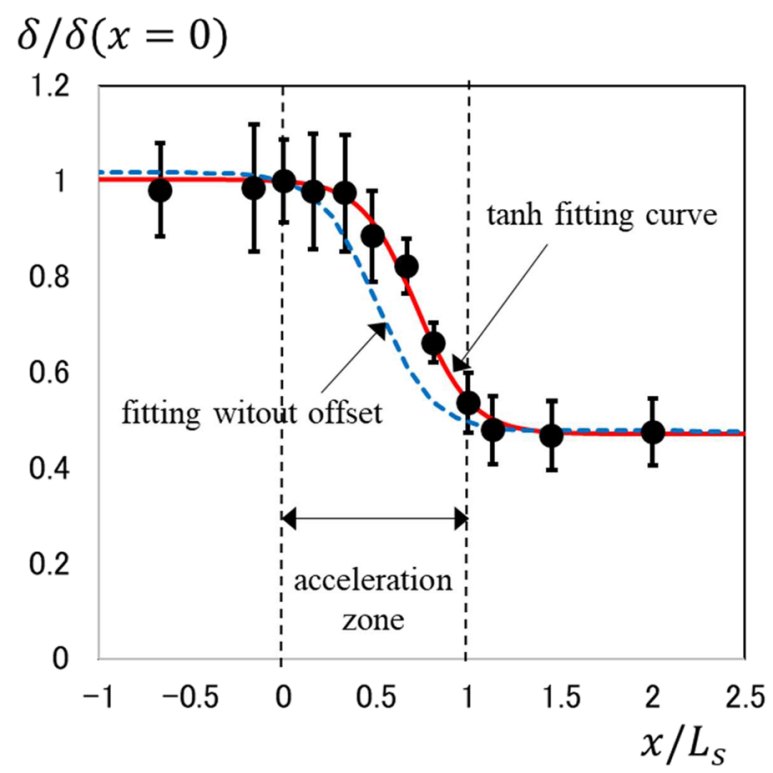

FIG. 9. Streamwise variation in $\delta$ measured by the fine DO probe. 
flow. These interesting tendencies can be theoretically explained using Eq. (27). The term $A \delta$ indicates the contribution of the mean free-surface velocity $U_{s}$ and the streamwise gradient $\partial U_{s} / \partial x$, and the term $B \delta^{-1}$ indicates the balance of the molecular diffusion and the turbulent diffusion. That is, the spatial acceleration of $U_{s}$ and the growth of the turbulent diffusion lead to a streamwise reduction in $\delta$. The term $A \delta$ can be calculated using the mean velocity distribution measured by the PIV. To obtain $\partial \delta / \partial x$, the measured $\delta$ is fitted to the following hyperbolic tangent curve:

$$
\delta(x)=\frac{\delta_{1}+\delta_{2}}{2}-\frac{\delta_{1}-\delta_{2}}{2} \tanh \left(A_{\delta}\left(x-x_{\delta}\right)\right),
$$

where $\delta_{1}=0.032 \mathrm{~cm}, \delta_{2}=0.015 \mathrm{~cm}, A_{\delta}=0.045$, and $x_{\delta}=54 \mathrm{~cm}$. Since $D_{t}(\delta)$ is unknown, the term $B \delta^{-1}$ cannot be obtained using Eq. (29). By mathematically substituting the value of $\partial \delta / \partial x$ that was calculated using Eq. (38) and the $A \delta$ term calculated using Eqs. (28) and (27), the $B \delta^{-1}$ term can be evaluated.

Figure 10 shows the streamwise variations in the first term $-\mathrm{A} \delta$ and the second term $\mathrm{B} \delta^{-1}$ of Eq. (27). These two terms indicate the effects of the mean flow acceleration and the turbulent diffusion, respectively, as described above, and they are at nearly the same level upstream of the wedge entrance $\left(x / L_{s}=0\right)$. In addition, they both become remarkable in the acceleration zone, and they have negative peaks a bit downstream of the mid-position $\left(x / L_{s}=0.5\right)$. These terms most significantly contribute to the reduction in the CBT thickness near the inflectional point $\delta(x)$, as shown in Fig. 9. Although $B \delta^{-1}$ is always larger than $-A \delta$ in the whole region in the acceleration zone, the effect of the mean velocity acceleration was found to be comparable with that of the turbulent diffusion. Note that both $-\mathrm{A} \delta$ and $\mathrm{B} \delta^{-1}$ are still effective at the exit of the acceleration zone $\left(x / L_{s}=1\right)$, which corresponds to the fact that $\delta(x)$ continues to decrease here, as shown in Fig. 9. The term $B \delta^{-1}$ is always negative in the acceleration zone. This fact and the definition of $B \delta^{-1}$ in Eq. (29) imply

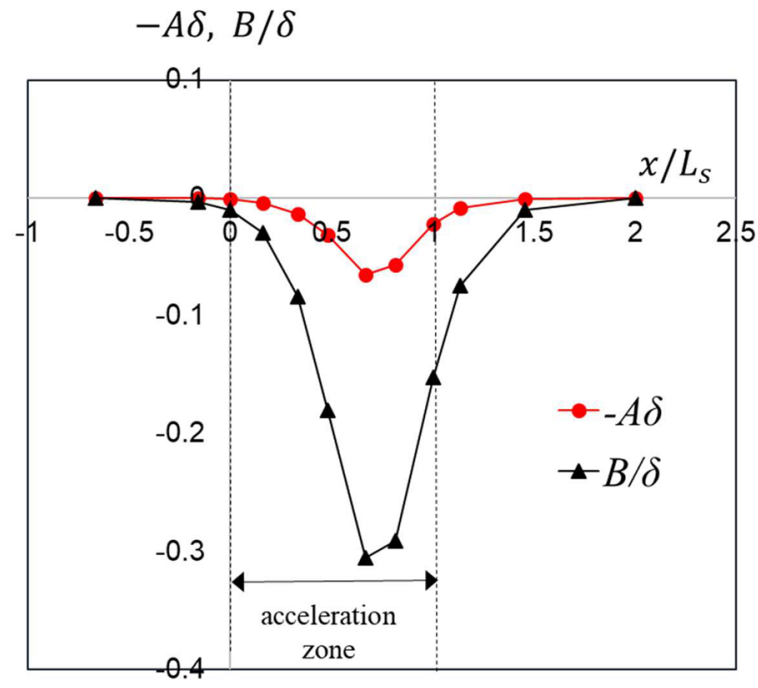

FIG. 10. Streamwise variations in the mean flow acceleration term $-A \delta$ and the turbulent diffusion term $B \delta^{-1}$. that turbulence diffusion is larger than the molecular diffusion. With the velocity acceleration, the free-surface turbulence and the related concentration transfer are expected to be promoted. The decrease in $\delta(x)$ makes the absolute value of $B \delta^{-1}$ larger. In contrast, when the CBT reduces further, $D_{t}(\delta)$ becomes close to zero and is on the same order of the constant molecular diffusion coefficient, $D$. Thus, $B \delta^{-1}$ becomes smaller, resulting in the appearance of the negative peak.

\section{Relaminarization in open-channel acceleration}

The gradual reduction in the cross-sectional area increases the mean velocity in the streamwise direction, as shown in Fig. 6. Even though the turbulent statistics also become larger with the mean flow acceleration, the increase in the turbulence is relatively small compared with that in the mean flow during the acceleration process. Consequently, the non-dimensional turbulent statistics normalized by the characteristic velocity scale decreases due to the mean flow acceleration, as does the frequency of the large-scale vortex occurrence. This physical phenomenon is called relaminarization. Relaminarization in duct flow is observed due to several physical causes, ${ }^{40,49}$ including turbulent dissipation due to viscosity and turbulent energy consumption due to the external forces.

To examine the occurrence of relaminarization in the present accelerated flow in an open channel, Fig. 11 shows the longitudinal distribution of the normalized free-surface turbulent intensity $u_{s}{ }^{\prime} / U_{s}$. The experimental results of Narayanan and Ramjee ${ }^{39}$ are also included for comparison; here, the maximum turbulent intensity in each section $u_{\text {max }}^{\prime}$ is plotted. The flat-bottom zone situated upstream of the acceleration has a value of 0.075 , and thereafter, this value decreases to 0.05 due to the acceleration. The normalized factor, on the order of $10^{-2}$, decreases in the acceleration zone. Even though it is generally difficult to define relaminarization and the criterion

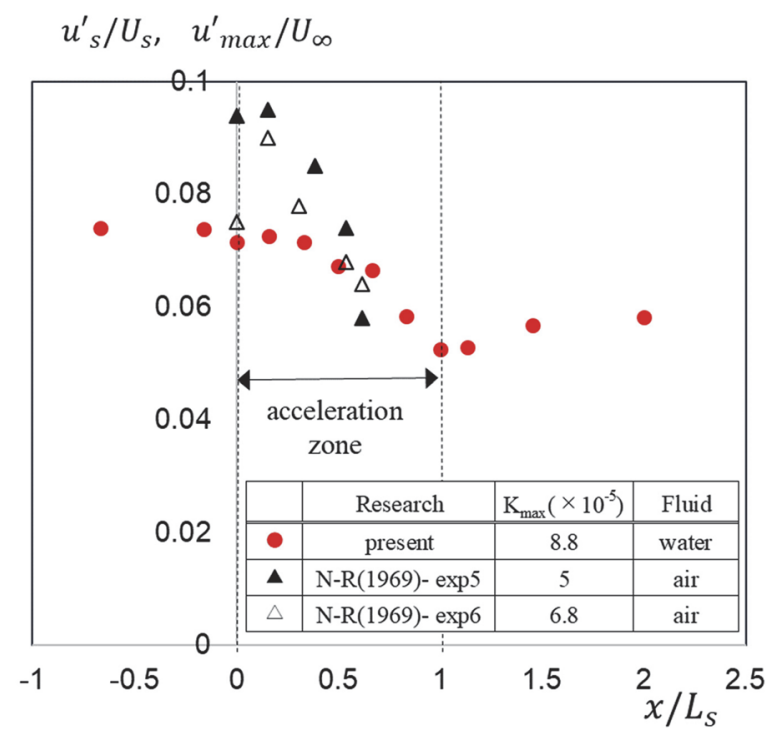

FIG. 11. Streamwise distribution of the normalized free-surface turbulence intensity $u_{s}^{\prime} / U_{s}$. 
for its onset is not standardized, a velocity fluctuation relative to the mean velocity is observed in the presented experiment. Therefore, this study concluded that the flow condition shifts toward laminar behavior through the acceleration zone.

The generation term in the $2 \mathrm{D}$ turbulent kinetic energy equation is given by $G(x, y) \equiv-\overline{u u} \frac{\partial U}{\partial x}-\overline{u v} \frac{\partial U}{\partial y}$. In the steady uniform unidirectional flow with no spatial acceleration, the first term becomes zero, and only the second term is considered. In contrast, the accelerated flow makes the first term more significant. Except for the part very near the bottom, the first term becomes negative since $\partial U / \partial x>0$, and the turbulence generation is reduced. Particularly, near the free surface, the second term becomes almost zero and lacks turbulence energy even in the uniform flow. In the accelerated flow, the total turbulence generation $G$ is negative, and the turbulence is smaller than that estimated by the free-surface velocity. Hence, it is very difficult to predict the gas transfer velocity and the related turbulence intensity in such local acceleration zones.

Furthermore, the downward currents under the free-surface are expected to be smaller over the wedge compared with those observed in the horizontal bottom section. It results in strengthening of the SAVS as mentioned above, which may be reduced. Hence, it may contribute to the relaminarization in the acceleration zone.

\section{Local gas transfer in an accelerated open channel}

The free-surface velocity begins to increase at the entrance of the acceleration zone, followed by the free-surface turbulent statistics, such as the turbulent kinetic energy and the SD intensity. The streamwise reduction in the thickness of the CBT $\delta$ begins not at the wedge edge $(x=0)$, but in the acceleration zone; this is also true for the free-surface turbulence. The gas transfer velocity $k_{L}$, which is proportional to the inverse of $\delta$, is expected to be remarkably elevated at the mid-point of the acceleration zone and downstream $\left(x / L_{s}>0.5\right)$, where the effects of both the mean velocity acceleration and the free-surface turbulent growth are significant.

Figure 12 shows the streamwise variation in the nondimensional local gas transfer velocity normalized by the square root of the free-surface turbulent kinetic energy $k_{L} / k_{s}^{1 / 2}$. This factor becomes 1.5 times that in the flat-bottom zone $(x<0)$. This may be caused by the physical effect peculiar to the streamwise acceleration, i.e., $-A \delta$, which increases the ratio of the gas transfer velocity to the free-surface turbulence. The standard SD model, ${ }^{22}$ which evaluates the gas transfer velocity using the surface divergence, is given by

$$
k_{L}=\alpha \sqrt{D \beta^{\prime}},
$$

where $\alpha$ is a proportionality coefficient ranging from 0.1 to $0.7 .^{26}$

Figure 12 also shows the streamwise variation in $\alpha$. The present data vary within the range of the values obtained by the previous studies, including the studies on oscillation tanks and wind-shear currents. Most importantly, the streamwise variation increases in the acceleration zone, which implies that the gas is more excessively transferred in the uniform flow without streamwise acceleration. The normalized gas transfer rate, $k_{L} / k_{s}^{1 / 2}$, and $\alpha$ slightly decrease in the shallow flat zone $\left(x / L_{s}>1.0\right)$ where the flow acceleration $\partial U / \partial x$ is not expected to contribute to the gas transfer. The turbulence diffusion near the free surface plays a key role there. In particular,

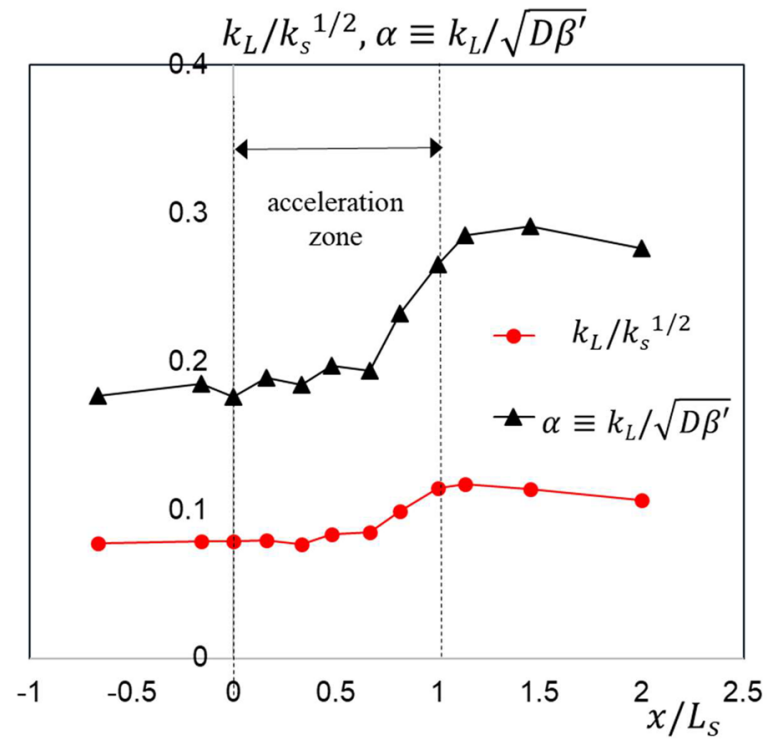

FIG. 12. Streamwise variation in non-dimensional local gas transfer $k_{L} / k_{s}^{1 / 2}$ and proportional coefficient of the SD model $\alpha$.

the difference between the molecular diffusion and turbulence diffusion at the CBT depth controls the growth of the CBT. Additionally, the updated CBT is fed back to $D_{t}(\delta)$; thus, we must consider their physical interactions.

At a sufficient downstream distance from the exit of the acceleration zone, the CBT is expected to be stable, i.e., $\partial \delta / \partial x=0$. Although it is not hard to examine this assumption in our laboratory flume with the finite length, this can be explained reasonably using the present CBT theory in the following way. We are able to only focus on the second term in the right-hand-side of Eq. (27), $B \delta^{-1}$, in the uniform flow without streamwise acceleration. Figure 13 shows a phenomenological model referring relationship between the vertical profiles of the molecular and turbulence diffusion coefficients. In this discussion, for convenience, $D$ and $D_{t}$ were expressed including the coefficients $1-1 / e$ and $1 / e$. It is generally considered that the turbulence diffusion is much more remarkable than the molecular diffusion $\left(D_{t}>D\right)$, except for near the boundaries of the bottom and the free surface in the case of open-channel turbulence. In contrast, the turbulence diffusion with the vertical direction becomes zero $\left(D_{t}=0\right)$ at the flat free surface. This implies that the $\left(D_{t}\right.$ $<D)$ layer exists very close to the free surface. In the case that the CBT thickness $\delta$ is within the layer where the molecular diffusion is more influential than the turbulence diffusion $\left(D_{t}<D\right)$, as shown in Fig. 13(a), the positive $B \delta^{-1}$ induces an increase in $\delta$ with the downstream. At the same time, the concentration gradient $\partial C / \partial y$ is also reduced. As the streamwise variation in the turbulent concentration flux $-\overline{c v}$ is assumed to be small, Eq. (24) allows an increase in $D_{t}$ near the free surface with a decrease in $\partial C / \partial y$. After further traveling downstream, the depth of the CBT moves in the layer in which the turbulence diffusion is significant, as shown in Fig. 13(b). Then, $\delta$ starts to decrease downstream due to $B \delta^{-1}<0$, and the increase in $\partial C / \partial y$ returns the situation to that of Fig. 13(a) in which the 


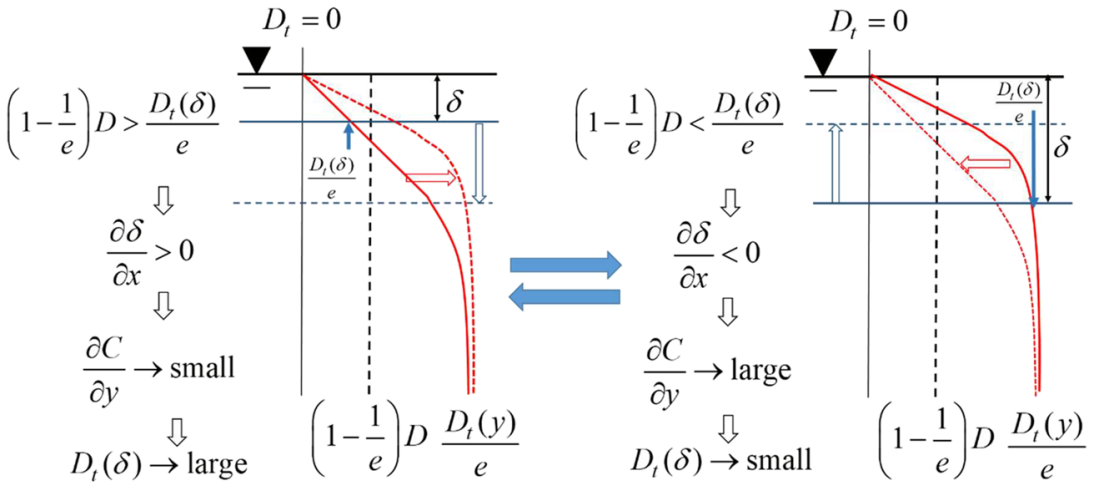

(b)
CBT depth is in the layer of $\left(D_{t}<D\right) . \delta$ is expected to converge the repeating of the two stages of Figs. 13(a) and 13(b) when traveling downstream.

\section{CONCLUSIONS}

This study focused on local gas transfer in an accelerated openchannel flow and conducted a theoretical expansion of the streamwise development of the CBT related to the gas transfer velocity. In addition, the CBT was evaluated using a local DO profile measured with an extra-fine needle probe. The mean velocity and turbulence were measured via a PIV system including a high-speed camera and an LLS. The newly proposed theoretical model and the measurement results enable us to understand how the CBT is formed and how it varies in the streamwise direction as influenced by the mean velocity acceleration and the production of free-surface turbulence. The main results are summarized below.

(1) The streamwise variation in the CBT was mathematically introduced by integrating the $2 \mathrm{D}$ gas-concentration transport equation from the free surface to the depth of the CBT. This theoretical model suggests that two types of factors, i.e., the streamwise acceleration of the free-surface mean flow $\partial U_{s} / \delta x$ and the ratio of the turbulent diffusivity at the depth of the CBT to the molecular diffusivity $D_{t}(\delta) / D$, significantly control the streamwise variation in the CBT $\partial \delta / \delta x$.

(2) The DO profile was measured at several test sections in an initially deaerated open channel. The DO values were obtained by traversing the probe in the vertical direction during the first-order recovery stage when the normalized dissolved concentration varied linearly. The streamwise variation in the CBT was evaluated by fitting an exponential function at each section.

(3) In the streamwise direction in the acceleration zone, the PIV results showed that the free-surface mean velocity and turbulence increase and that the cross-sectional area gradually decreases. In addition, the streamwise variations in $k_{s}$ and $\beta^{\prime}$ were small in the upstream side in the acceleration zone, which corresponds to the variation in the free-surface velocity. It was noticed that the free-surface turbulence factors started to increase not at the entrance, but around the mid-position within the acceleration zone. It is also of particular significance that they continued to increase at the exit, which implies that the reduced turbulence generation in the acceleration zone started to restore there. In addition, relaminarization occurred over the wedge, which is in agreement with the previous experimental studies.

(4) The comparison of the theoretical CBT model and the measurement data indicated that the contributions of both the mean velocity and the turbulence diffusion are significant in the acceleration zone. The CBT began to decrease a bit downstream of the entrance corresponding to the variation in the free-surface turbulence factors, and the inflectional point of $\delta(x)$ appeared at the downstream side of the acceleration zone and continued to decrease at the exit.

(5) The proportional coefficient of the SD model increased in the acceleration zone. This suggests that the gas was more excessively transferred by the mean velocity acceleration than by the uniform flow without the streamwise acceleration. The above-mentioned local analysis of the CBT is expected to be applied to air-water interfacial mass-heat transfer in non-uniform flows.

There have not been any detailed discussions in previous works on what drives the CBT in accelerated open-channel zones. By introducing the mathematical expression of the streamwise variation in CBT, this study not only suggested turbulence diffusion, but the mean flow gradient also significantly contributed to the gas transfer. The theoretically introduced terms, $-A \delta$ and $B \delta^{-1}$, can be obtained by the accurate measurement of $\delta$ with a sub-millimeter scale in a meter-scale laboratory flume. The obtained results and findings here could not be achieved before.

Generally speaking, the gas transfer velocity $k_{L}$ is often evaluated as a bulk-mean factor by many previous works on gas transfer in channel flows and oscillation tanks. Whereas in non-uniform flow fields, such as acceleration zones, secondary currents by sidewalls, wake behind structures and sedimentations, Langmuir circulation in ocean (e.g., Ref. 50), gas-convergence zone, and gasdivergence zone are expected to appear locally at the same time. The 
mechanism of local gas transfer and related modeling of $k_{L}$ were not known for sure. The present study may be an occasion to promote the investigation of such a local gas transfer phenomenon. Although this study focused on only one hydraulic condition, the physical mechanism of the streamwise formation of the CBT could be explained and discussed reasonably based on the combination of the newly proposed theoretical expression and accurate velocity and concentration measurements. The influences of the bottom configuration and roughness appear in the $U_{s}$ acceleration, $D_{t}$, and CBT, which are key variables in this theory. When the $U_{s}$ and CBT are measured as in the present experiments, this theory is applicable to the vertical two-dimensional open-channel turbulence without free surface fluctuations. Under these conditions, a similar discussion will be possible for other non-uniform fields, such as flows through submerged vegetation patches, over sand waves, and strip roughness. We aim to examine the limitations of the proposed theory in more detail together with further experimental studies. Furthermore, if a reliable evaluation of $D_{t}$ using the LIF or the eddy correlation method becomes possible, the streamwise growth and decay of the CBT can be predicted. Model improvements for violent free surface flows will also be significant in future work.

\section{ACKNOWLEDGMENTS}

The present study was carried out under the financial support from the Research Project Grants-In-Aid for Scientific Research (B) of Japanese Government (Kakenhi Grant No. 17H03311, Principal Investigator: M. Sanjou) and the Research Project GrantIn-Aid for Scientific Research (B) of Japanese Government (Kakenhi Grant No. 19H02249, Principal Investigator: Y. Sugihara). The authors gratefully acknowledge them and Mr. Ryosuke Nobuhara and Mr. Kazuya Maeguchi for their support in laboratory and field works.

\section{NOMENCLATURE}

$\Delta C \equiv C_{s}-C_{b}$

$\alpha$

$\beta^{\prime}$

$\tilde{\beta}$

$\delta$

$\tilde{c}$

C

$C^{\prime} \equiv\left(C_{s}-C\right) /\left(C_{s}-C_{0}\right)$

$C_{b}$

$C_{s}$

D

$D_{t}$

$\mathrm{Fr} \equiv U_{m} / \sqrt{g H}$

$H$
$k_{L}$ $k_{s} \equiv\left(u_{s}{ }^{2}+w_{s}{ }^{2}\right) / 2$ $k_{2}$ K $L_{s}$ $\operatorname{Re} \equiv U_{m} H / v$

u

$u^{\prime}$

$\tilde{u}$

$u_{s}^{\prime}$

U

$U_{s}$

$v$

$v^{\prime}$

$\tilde{v}$

V

$w$

$w^{\prime}$

$\tilde{w}$

$w_{s}^{\prime}$

W

$x$

$y$

$y^{\prime}$

$z$

intensity of the surface velocity divergence evaluated by rms operation

instantaneous surface velocity divergence thickness of the concentration boundary layer (CBT)

instantaneous dissolved oxygen concentration

dissolved oxygen concentration

normalized dissolved oxygen concentration

bulk dissolved oxygen concentration

saturated dissolved oxygen concentration diffusion coefficient of the dissolved oxygen

turbulent diffusion coefficient of the dis-

solved oxygen

Froude number

water depth gas transfer velocity

turbulent kinetic energy at the free surface reaeration coefficient

pressure gradient parameter

streamwise length of the acceleration section

Reynolds number using the bulk-mean veloc-

ity

turbulence component in the streamwise direction

turbulence intensity in the streamwise direction

instantaneous velocity component in the streamwise direction

streamwise turbulence intensities in the free surface

time-averaged velocity in the streamwise direction

time-averaged streamwise velocity at the free surface

turbulence component in the vertical direction

turbulence intensity in the vertical direction instantaneous velocity component in the vertical direction

time-averaged velocity in the vertical direction

turbulence component in the spanwise direction

turbulence intensity in the spanwise direction instantaneous velocity component in the spanwise direction

spanwise turbulence intensities in the free surface

time-averaged velocity in the spanwise direction

streamwise coordinate

upward vertical coordinate with the bottom origin

downward vertical coordinate with the freesurface origin

spanwise coordinate

\section{APPENDIX: DERIVATIONS OF EQUATIONS}

Derivations of equations in the main body are indicated here. How to lead Eq. (12) is as follows:

$$
\begin{aligned}
\int_{0}^{\delta} V \frac{\partial C}{\partial y} d y & =\int_{0}^{\delta}\left(-\int_{0}^{y} \frac{\partial U}{\partial x} d y\right) \frac{\partial C}{\partial y} d y \\
& =-\left[C \int_{0}^{y} \frac{\partial U}{\partial x} d y\right]_{0}^{\delta}+\int_{0}^{\delta} C \frac{\partial U}{\partial x} d y \\
& =-C(\delta) \int_{0}^{\delta} \frac{\partial U}{\partial x} d y+C(0) \int_{0}^{0} \frac{\partial U}{\partial x} d y+\int_{0}^{\delta} C \frac{\partial U}{\partial x} d y \\
& =-C(\delta) \frac{\partial U_{s}}{\partial x} \delta+\int_{0}^{\delta} C \frac{\partial U}{\partial x} d y
\end{aligned}
$$


How to lead Eq. (18) is as follows:

$$
\begin{aligned}
\int_{0}^{\delta} C d y & =\int_{0}^{\delta} e^{-\frac{y}{\delta}} \Delta C d y+\int_{0}^{\delta} C_{b} d y \\
& =\Delta C \int_{0}^{\delta} e^{-\frac{y}{\delta}} d y+\left[C_{b} y\right]_{0}^{\delta} \\
& =\Delta C\left[-\delta e^{-\frac{y}{\delta}}\right]_{0}^{\delta}+C_{b} \delta \\
& =\Delta C\left[-\delta e^{-1}+\delta\right]+C_{b} \delta \\
& =\delta\left(-\frac{\Delta C}{e}+\Delta C+C_{b}\right) .
\end{aligned}
$$

How to lead Eq. (19) is as follows:

$$
\begin{aligned}
\int_{0}^{\delta} U \frac{\partial C}{\partial x} d y & \approx U_{s} \int_{0}^{\delta} \frac{\partial C}{\partial x} d y \\
& =U_{s} \frac{\partial}{\partial x} \int_{0}^{\delta} C d y \\
& =U_{s} \frac{\partial}{\partial x}\left[\delta\left\{\Delta C\left(1-\frac{1}{e}\right)+C_{b}\right\}\right] \\
& =U_{s}\left\{\Delta C\left(1-\frac{1}{e}\right)+C_{b}\right\} \frac{\partial \delta}{\partial x} .
\end{aligned}
$$

How to lead Eq. (20) is as follows:

$$
\begin{aligned}
\int_{0}^{\delta} y e^{-\frac{y}{\delta}} d y & =\left[-y \delta e^{-\frac{y}{\delta}}\right]_{0}^{\delta}-\int_{0}^{\delta}-\delta e^{-\frac{y}{\delta}} d y \\
& =-\delta^{2} e^{-1}+\delta \int_{0}^{\delta} e^{-\frac{y}{\delta}} d y \\
& =-\frac{\delta^{2}}{e}+\delta\left[-\delta e^{-\frac{y}{\delta}}\right]_{0}^{\delta} \\
& =-\frac{\delta^{2}}{e}+\delta\left(-\delta e^{-1}+\delta\right) \\
& =-\frac{\delta^{2}}{e}+\delta^{2}\left(-\frac{1}{e}+1\right) \\
& =-\frac{2 \delta^{2}}{e}+\delta^{2}=\delta^{2}\left(1-\frac{2}{e}\right) .
\end{aligned}
$$

How to lead Eq. (21) is as follows:

$$
\begin{aligned}
\int_{0}^{\delta} C \frac{\partial U}{\partial x} d y & \approx\left[C\left(\frac{\partial U_{s}}{\partial x} y\right)\right]_{0}^{\delta}-\int_{0}^{\delta} \frac{\partial C}{\partial y} \frac{\partial U_{s}}{\partial x} y d y \\
& =C(\delta) \frac{\partial U_{s}}{\partial x} \delta-\frac{\partial U_{s}}{\partial x} \int_{0}^{\delta} y\left(-\frac{\Delta C}{\delta} e^{-\frac{y}{\delta}}\right) d y \\
& =\left(\frac{\Delta C}{e}+C_{b}\right) \frac{\partial U_{s}}{\partial x} \delta+\frac{\partial U_{s}}{\partial x} \frac{\Delta C}{\delta} \int_{0}^{\delta} y e^{-\frac{y}{\delta}} d y \\
& =\left(\frac{\Delta C}{e}+C_{b}\right) \frac{\partial U_{s}}{\partial x} \delta+\frac{\partial U_{s}}{\partial x} \Delta C\left(1-\frac{2}{e}\right) \delta \\
& =\frac{\partial U_{s}}{\partial x} \delta\left(\frac{\Delta C}{e}+C_{b}+\Delta C-\frac{2 \Delta C}{e}\right) \\
& =\frac{\partial U_{s}}{\partial x} \delta\left\{\Delta C\left(1-\frac{1}{e}\right)+C_{b}\right\}
\end{aligned}
$$

How to lead Eq. (22) is as follows:

$$
\begin{aligned}
\int_{0}^{\delta}( & \left.U \frac{\partial C}{\partial x}+V \frac{\partial C}{\partial y}\right) d y \\
= & U_{s}\left\{\Delta C\left(1-\frac{1}{e}\right)+C_{b}\right\} \frac{\partial \delta}{\partial x}+\frac{\partial U_{s}}{\partial x}\left\{\Delta C\left(1-\frac{1}{e}\right)+C_{b}\right\} \delta \\
& -\left(\frac{\Delta C}{e}+C_{b}\right) \frac{\partial U_{s}}{\partial x} \delta \\
= & U_{s}\left\{\Delta C\left(1-\frac{1}{e}\right)+C_{b}\right\} \frac{\partial \delta}{\partial x}+\frac{\partial U_{s}}{\partial x}\left(1-\frac{2}{e}\right) \Delta C \delta .
\end{aligned}
$$

How to lead Eq. (25) is as follows:

$$
\begin{aligned}
\int_{0}^{\delta}-\frac{\partial \overline{c v}}{\partial y} d y & =\int_{0}^{\delta} \frac{\partial}{\partial y}\left(D_{t} \frac{\partial C}{\partial y}\right) d y \\
& =\int_{0}^{\delta} \frac{\partial}{\partial y}\left(-D_{t} \frac{\Delta C}{\delta} e^{-\frac{y}{\delta}}\right) d y=-\frac{\Delta C}{\delta} \int_{0}^{\delta} \frac{\partial}{\partial y}\left(D_{t} e^{-\frac{y}{\delta}}\right) d y \\
& =-\frac{\Delta C}{\delta}\left[D_{t} e^{-\frac{y}{\delta}}\right]_{0}^{\delta}=-\frac{\Delta C}{\delta}\left\{D_{t}(\delta) e^{-1}-D_{t}(0) e^{0}\right\} \\
& =-\frac{\Delta C}{\delta e} D_{t}(\delta) .
\end{aligned}
$$

\section{DATA AVAILABILITY}

The data that support the findings of this study are available from the corresponding author upon reasonable request.

\section{REFERENCES}

'E. C. Tsivoglou, R. L. O'Connel, C. M. Walter, P. J. Godsil, and G. S. Logsdon, "Tracer measurements of atmospheric reaeration: I. laboratory studies," J.-Water Pollut. Control Fed. 37, 1343-1362 (1965), available at https://www.jstor.org/stable/25035386?seq=19\#metadata_info_tab_contents.

${ }^{2}$ B. J. Copeland and W. R. Duffer, "Use of a clear plastic dome to measure gaseous diffusion rates in natural waters," Limnol. Oceanogr. 9, 494-499 (1964).

${ }^{3}$ D. Vachon, Y. T. Prairie, and J. J. Cole, "The relationship between near-surface turbulence and gas transfer velocity in freshwater systems and its implications for floating chamber measurements of gas exchange," Limnol. Oceanogr. 55, 17231732 (2010).

${ }^{4}$ S. C. Chapra and D. M. Di Toro, "Delta method for estimating primary production, respiration and reaeration in streams," J. Environ. Eng. 117, 640-655 (1991).

${ }^{5}$ G. B. McBride and S. C. Chapra, "Rapid calculation of oxygen in streams: Approximate delta method," J. Environ. Eng. 131, 336-342 (2005).

${ }^{6} \mathrm{M}$. Sanjou, T. Okamoto, and I. Nezu, "Dissolved oxygen transfer into a square embayment connected to an open-channel flow," Int. J. Heat Mass Transfer 125, 1169-1180 (2018).

${ }^{7}$ N. Morse, W. B. Bowden, A. Hackman, C. Pruden, E. Steiner, and E. Berger, "Using sound pressure to estimate reaeration in streams," J. North Am. Benthological Soc. 26, 28-37 (2007).

${ }^{8} \mathrm{~N}$. Herlina and G. H. Jirka, "Experiments on gas transfer at the air-water interface induced by oscillating grid turbulence," J. Fluid Mech. 594, 183-208 (2008).

${ }^{9}$ E. A. Variano and E. A. Cowen, "Turbulent transport of a high-Schmidt-number scalar near an air-water interface," J. Fluid Mech. 731, 259-287 (2013).

${ }^{10}$ D. J. O'Connor and W. E. Dobbins, "Mechanism of reaeration in natural stream," Trans., ASCE 123, 641-666 (1958), available at https://cedb.asce.org/ CEDBsearch/record.jsp? dockey $=0294423$.

${ }^{11}$ S. Komori, Y. Murakami, and H. Ueda, "The relationship between surfacerenewal and bursting motions in an open-channel flow," J. Fluid Mech. 203, 103-123 (1989). 
${ }^{12}$ R. G. Jackson, "Sedimentological and fluid-dynamic implications of the turbulent bursting phenomenon in geophysical flows," J. Fluid Mech. 77, 531-560 (1976).

${ }^{13}$ S. Komori, H. Ueda, F. Ogino, and T. Mizushina, "Turbulence structure and transport mechanism at the free surface in an open channel flow," Int. J. Heat Mass Transfer 25, 513-521 (1982).

${ }^{14}$ S. Kumar, R. Gupta, and S. Banerjee, "An experimental investigation of the characteristics of free-surface turbulence in channel flow," Phys. Fluids 10, 437 (1998).

${ }^{15}$ R. A. Handler, T. F. Swean, R. I. Leighton, and J. D. Swearingen, "Length scales and the energy balance for turbulence near a free surface," AIAA J. 31, 1998 (1993).

${ }^{16} \mathrm{R}$. Nagaosa, "Direct numerical simulation of vortex structures and turbulent scalar transfer across a free surface in a fully developed turbulence," Phys. Fluids 11, 1581-1595 (1998).

${ }^{17} \mathrm{M}$. Brocchini and D. H. Peregrine, "The dynamics of strong turbulence at free surfaces. Part 1. Description,” J. Fluid Mech. 449, 225-254 (2001).

${ }^{18}$ C. C. Chickadel, A. R. Horner-Devine, S. A. Talke, and A. T. Jessup, "Vertical boil propagation from a submerged estuarine sill,” Geophys. Res. Lett. 36, L10601, https://doi.org/10.1029/2009gl037278 (2009).

${ }^{19}$ T. L. Mandel, S. Gakhar, H. Chung, I. Rosenzweig, and J. R. Koseff, "On the surface expression of a canopy-generated shear instability," J. Fluid Mech. 867, 633-660 (2019).

${ }^{20} \mathrm{~J}$. S. Gulliver and M. J. Halverson, "Air-water gas transfer in open channels," Water Resour. Res. 25, 1783-1793, https://doi.org/10.1029/wr025i008p01783 (1989).

${ }^{21}$ D. B. Moog and G. H. Jirka, "Air-water gas transfer in uniform channel flow," J. Hydraul. Eng. 125(1), 3-10 (1999).

${ }^{22}$ M. J. McCready, E. Vassiliadou, and T. J. Hanratty, "Computer simulation of turbulent mass transfer at a mobile interface," AIChE J. 32, 1108-1115 (1986).

${ }^{23} \mathrm{~A}$. Tamburrino and J. S. Gulliver, "Free-surface turbulence and mass transfer in a channel flow," AIChE J. 48, 2732-2743 (2002).

${ }^{24}$ S. Banerjee, D. Lakehal, and M. Fulgosi, "Surface divergence models for scalar exchange between turbulent streams," Int. J. Multiphase Flow 30, 963-977 (2004).

${ }^{25} \mathrm{~J}$. Magnaudet and I. Calmet, "Turbulent mass transfer through a flat shear free surface," J. Fluid Mech. 553, 155-185 (2006).

${ }^{26} \mathrm{M}$. Sanjou, I. Nezu, and T. Okamoto, "Surface velocity divergence model of air/water interfacial gas transfer in open-channel flows," Phys. Fluids 29, 045107 (2017).

${ }^{27}$ A. Leman, A. M. Holland, and R. O. Tinoco, "Identifying the dominant physical processes for mixing in full-scale raceway tanks," Renewable Energy 129, 616-628 (2018).

${ }^{28}$ A. J. Szeri, "Boundary layers at a dynamic interface: Air-sea exchange of heat and mass," J. Geophys. Res.: Oceans 122, 2781-2794, https://doi.org/10.1002/ 2016jc012312 (2018).

${ }^{29} \mathrm{C}$.-Y. Tseng and R. O. Tinoco, "A model to predict surface gas transfer rate in streams based on turbulence production by aquatic vegetation," Adv. Water Resour. 143, 103666 (2020).

${ }^{30}$ P. A. Durbin and B. A. Pettersson Reif, Statistical Theory and Modeling for Turbulent Flows (John Wiley \& Sons, 2011).
${ }^{31}$ S. Corrsin, "Limitations of gradient transport models in random walks and in turbulence," Adv. Geophys. 18A, 25-60 (1974), available at https://www. sciencedirect.com/science/article/abs/pii/S0065268708604513.

${ }^{32}$ F. Hamba, "Nonlocal expression for scalar flux in turbulent shear flow," Phys. Fluids 16, 1493 (2004).

${ }^{33}$ B. Jahne and H. Haubecker, “Air water gas exchange," Annu. Rev. Fluid Mech. 30, 443-468 (1998).

${ }^{34}$ W. K. Lewis and W. G. Whitman, "Principles of gas absorption," Ind. Eng. Chem. 16, 1215-1220 (1924).

${ }^{35}$ S. P. McKenna and W. R. McGillis, "The role of free-surface turbulence and surfactants in air-water gas transfer," Int. J. Heat Mass Transfer 47, 539-553 (2004).

${ }^{36}$ J. G. Wissink, H. Herlina, Y. Akar, and M. Uhlmann, "Effect of surface contamination on interfacial gas transfer," J. Fluid Mech. 830, 5-34 (2017).

${ }^{37}$ I. Nezu and M. Sanjou, "PIV and PTV measurements in hydro-sciences with focus on turbulent open-channel flows," J. Hydro-Environ. Res. 5, 215-230 (2010).

${ }^{38} \mathrm{M}$. Sanjou and I. Nezu, "Hydrodynamic characteristics and related masstransfer properties in open-channel flows with rectangular embayment zone," Environ. Fluid Mech. 13, 527-555 (2013).

${ }^{39}$ M. A. B. Narayanan and V. Ramjee, "On the criteria for reverse transition in a two-dimensional boundary layer flow," J. Fluid Mech. 35, 225-241 (1969).

${ }^{40}$ R. Ranjan and R. Narasimha, "An assessment of the two-layer quasi-laminar theory of relaminarization through recent high-Re accelerated TBL experiments," J. Fluids Eng. 139, 111205 (2017).

${ }^{41}$ R. F. Blackwelder and L. S. G. Kovasznay, "Large-scale motion of a turbulent boundary layer during relaminarization," J. Fluid Mech. 53, 61-83 (1972).

${ }^{42}$ D. Warnack and H. H. Fernholz, "The effects of a favourable pressure gradient and of the Reynolds number on an incompressible axisymmetric turbulent boundary layer. Part 2. The boundary layer with relaminarization," J. Fluid Mech. 359, 357-381 (1998).

${ }^{43}$ M. P. Escudier, A. Abdel-Hameed, M. W. Johnson, and C. J. Sutcliffe, "Laminarisation and re-transition of a turbulent boundary layer subjected to favourable pressure gradient," Exp. Fluids 25, 491-502 (1998).

${ }^{44}$ C. Bourassa and F. O. Thomas, "An experimental investigation of a highly accelerated turbulent boundary layer," J. Fluid Mech. 634, 359-404 (2009).

${ }^{45}$ P. M. Morreti and W. M. Kays, "Heat transfer to a turbulent boundary layer with varying free-stream velocity and varying surface temperature-An experimental study," Int. J. Heat Mass Transfer 8, 1187-1202 (1965).

${ }^{46}$ S. J. Kline, W. C. Reynolds, F. A. Schraub, and P. W. Runstadler, "The structure of turbulent boundary layers," J. Fluid Mech. 30, 741-773 (1967).

${ }^{47}$ Z. Chen, C. Jiang, and H. Nepf, "Flow adjustment at the leading edge of a submerged aquatic canopy," Water Resour. Res. 49, 5537-5551, https://doi.org/10.1002/wrcr.20403 (2013).

${ }^{48} \mathrm{H}$. Herlina and J. G. Wissink, "Simulation of air-water interfacial mass transfer driven by high-intensity isotropic turbulence," J. Fluid Mech. 860, 419-440 (2019).

${ }^{49}$ R. Narasimha and K. R. Sreenivasan, "Relaminarization of fluid flows," Adv. Appl. Mech. 19, 221-309 (1979).

${ }^{50} \mathrm{M}$. Sanjou and I. Nezu, "Secondary current properties generated by windinduced water waves in experimental conditions," Adv. Oceanogr. Limnol. 5, 1-17 (2014). 